\title{
Wind and turbulence measurements by the Middle and Upper Atmosphere Radar (MUR): comparison of techniques
}

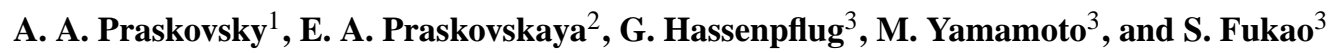 \\ ${ }^{1}$ National Center for Atmospheric Research, Boulder, Colorado, USA \\ ${ }^{2}$ Colorado Research Associates, Boulder, Colorado, USA \\ ${ }^{3}$ Radio Science Center for Space and Atmosphere, Kyoto University, Kyoto, Japan
}

Received: 18 September 2003 - Revised: 7 April 2004 - Accepted: 5 May 2004 - Published: 29 November 2004

Part of Special Issue "10th International Workshop on Technical and Scientific Aspects of MST Radar (MST10)"

\begin{abstract}
The structure-function-based method (referred to as UCAR-STARS), a technique for estimating mean horizontal winds, variances of three turbulent velocity components and horizontal momentum flux was applied to the Middle and Upper atmosphere Radar (MUR) operating in spaced antenna (SA) profiling mode. The method is discussed and compared with the Holloway and Doviak (HAD) correlationfunction-based technique. Mean horizontal winds are estimated with the STARS and HAD techniques; the Doppler Beam Swinging (DBS) method is used as a reference for evaluating the SA techniques. Reasonable agreement between SA and DBS techniques is found at heights from $5 \mathrm{~km}$ to approximately $11 \mathrm{~km}$, where signal-to-noise ratio was rather high. The STARS and HAD produced variances of vertical turbulent velocity are found to be in fair agreement. They are affected by beam-broadening in a different way than the DBS-produced spectral width, and to a much lesser degree. Variances of horizontal turbulent velocity components and horizontal momentum flux are estimated with the STARS method, and strong anisotropy of turbulence is found. These characteristics cannot be estimated with correlation-function-based SA methods, which could make UCAR-STARS a useful alternative to traditional SA techniques.
\end{abstract}

Key words. Radio science (remote sensing; signal processing; instruments and techniques)

\section{Introduction}

During the last four decades, spaced antenna (SA) methods for measuring characteristics of a scattering medium at altitudes from the low troposphere to the upper mesosphere and

Correspondence to: A. A. Praskovsky

(praskov@ucar.edu) ionosphere have become widely used (e.g. Fooks, 1965; Fedor, 1967; Manson et al., 1974; Röttger and Vincent, 1978; Vincent and Röttger, 1980; Meek, 1980a; Röttger, 1981; Vincent et al., 1987; Larsen and Röttger, 1989; Riggin et al., 1997; Cohn et al., 1997, 2001; and many others). Although measurements were mainly focused on mean horizontal winds, other parameters, such as variance of vertical turbulent velocity and spatial scales of refractive index irregularities, have also been measured (e.g. Brown et al., 1995a, b; Hall et al., 1998; and Chau and Balsley, 1998).

The basic concept of the SA approach is presented in the classic paper by Briggs et al. (1950), and a diversity of SA methods has been developed (for reviews see, e.g. Hocking et al., 1989; Fukao and Palmer, 1991; and Palmer, 1994). The methods could be divided into two clearly distinguishable groups in accordance with the different approaches to considering received signals from multiple antennas (Doviak et al., 1996), and there are numerous specific techniques within each approach. The first approach assumes models of the diffraction pattern on the ground without relating the models to properties of a scattering medium. The techniques most used in this group are referred to as Full Correlation Analysis (FCA) in the time domain, and Full Spectral Analysis (FSA) in the frequency domain (Briggs, 1984; and Briggs and Vincent, 1992; respectively). This heuristical approach neither takes into account specific characteristics of the scattering medium nor of the radar parameters.

The second approach relates properties of the refractive index field and its advection flow to parameters of echoes in spaced receivers for a specific radar; therefore, models of the scattering medium are assumed. Liu et al. (1990) were the first to realize this approach; the Liu et al. theory was generalized and further expanded by Doviak et al. (1996), below referred to as DLH. The technique based on this approach is referred to below as the Holloway And Doviak (HAD) method; it is described in Holloway et al. (1997b), below referred to as HDC. 
As emphasized by many authors (e.g. Briggs and Vincent, 1992; Sheppard et al., 1993; Hocking et al., 1989), all SA methods are basically similar in that they utilize the same initial information: time series of amplitude and phase of signals from several receivers. The methods differ by (1) mathematical tools for analyzing multiple signals (auto and/or cross correlation functions; auto and/or cross spectra); (2) parameters of these functions to be estimated; (3) equations for relating these parameters to characteristics of the scattering medium; and (4) assumptions which are adopted for deriving the operational equations. Although basically similar, all methods produce important information about a scattering medium. Multiple signals from several receivers provide an enormous amount of raw information. Each data analysis tool (spectra, correlation functions, wavelets, etc.) extracts only a small fraction of useful information from multiple random signals; therefore, different techniques do not compete but rather complement each other.

A structure function (SF)-based approach to measuring characteristics of a scattering medium by SA radars has been developed recently by Praskovsky and Praskovskaya (2003a, 2003b), below referred to as PPa and PPb, and the name UCAR-STARS was coined to describe the data analysis method based on this approach. The acronym "UCARSTARS" stands for the "University Corporation for Atmospheric Research - STructure function Analysis of Received Signals". The goal of this paper is to evaluate the potential of the method for measuring mean horizontal winds and turbulence characteristics with SA radars. Detailed quantitative analysis of the STARS's performance, analysis of measurement errors, and comparison with other instruments, such as rawinsonde, is planned for the near future; these issues are beyond the scope of this paper. As a test bed for the method, we chose the Middle and Upper atmosphere Radar (MUR), which is one of the most powerful and flexible MesosphereStratosphere-Troposphere (MST) radars in the world. In order to compare the STARS results to correlation function (CF)-based SA technique results, we have chosen HAD from a diversity of existing SA methods because, in our opinion, HAD is the most advanced and rigorous CF-based SA technique. Theoretical comparison of HAD with the Briggs (1984) FCA method can be found in Holloway et al. (1997a). As shown in PPa, equations for SF of any order $p \geq 2$ can be derived and applied to practical measurements. On the contrary, only the second-order CF have been used in SA methods, and spectra are second-order functions as well. To compare STARS and HAD techniques, cross and auto SF at only $p=2$ are considered in this paper.

We found that at a sufficiently high signal-to-noise ratio (SNR) the STARS-produced mean horizontal winds are in reasonable agreement with those produced by HAD and the Doppler Beam Swinging (DBS) technique; the latter has been chosen as a reference for evaluating SA techniques. No independent wind measurements, such as by rawinsonde, were available during the experiment for evaluating SA techniques. Limited testing of the HAD technique was accomplished in the atmospheric boundary layer at very high SNR
(Cohn et al., 1997), but its performance for the MUR over heights above $5 \mathrm{~km}$ has not been thoroughly evaluated. We needed some kind of "independent true device" for comparing SA techniques, and DBS was chosen as such a "truth". Although DBS is not necessary accurate due to rather restrictive underlying assumptions, it is widely accepted by the atmospheric radar community as a reliable technique for measuring mean winds. The method has been extensively and thoroughly tested over several decades with independent instruments, such as rawinsonde, and its performance was found to be from "good" to "excellent" in most conditions (e.g. Steinhagen et al., 1994; Thomas et al., 1997; Kishore et al., 2000; Stankov et al., 2003; and references therein). In particular, Luce et al. (2001) reported "very good agreement" between DBS and radiosonde-measured mean winds for the MUR.

We found fair agreement between the STARS and HADproduced variances of vertical turbulent velocity. The SA results are affected by beam-broadening in a different way than the DBS-produced spectral width, and to a much lesser degree. We also present the STARS-measured variances of horizontal turbulent velocities and horizontal momentum flux. Strong anisotropy of turbulence is found, although caution is needed in the interpretation of the results. We have derived theoretical relations between CF and SF-based SA techniques. We have shown further that $\mathrm{CF}$ and SF-based techniques "sense" different physical features of a scattering medium and different temporal scales of the features, in spite of being related to one another. The major consequence of these differences is that variances of horizontal turbulent velocities and horizontal momentum flux can be potentially estimated with SF-based methods, while they cannot be estimated with CF-based methods. Therefore, UCAR-STARS could become a useful alternative to the traditional CF and spectra-based SA techniques.

\section{Estimating winds and turbulence}

In this section we summarize the basic equations for estimating the mean horizontal winds and turbulence characteristics with STARS and HAD techniques, as well as the assumptions which are adopted for deriving the equations. We also establish theoretical relations between the two SA techniques. Detailed derivation of the equations and discussion of the assumptions can be found in PPa, PPb, and DLH, HDC, respectively.

The transmitter of a SA profiling radar sends pulses of radio waves vertically upwards into the atmosphere and these are scattered by the refractive index irregularities to form a moving and changing diffraction pattern on the ground. Following PPa, the irregularities are referred to as scatterers independent of their physical nature. Therefore, the scatterer is defined as the refractive index irregularity scattering the transmitted waves of a specific frequency, and it is a property of the atmosphere to which the radar is sensitive. Following such a definition, each scatterer is characterized by 
its instantaneous location $\boldsymbol{x}_{i}(t)=\left\{x_{i}(t), y_{i}(t), z_{i}(t)\right\}$, velocity $\boldsymbol{W}_{i}(t)=\left\{U_{i}(t), V_{i}(t), W_{i}(t)\right\}$, and reflectivity $\Delta n_{i}(t)$. Hereafter $t$ is time, $i=1,2, \ldots, M$, and $M$ is a number of scatterers in the illuminated volume. The geophysical coordinate system with $\mathrm{z}$-axis directed upwards, $\mathrm{x}$-axis towards east, and $y$-axis towards north is used hereafter; the values in the brackets \{\} denote the Cartesian components of a vector.

The magnitude and phase of the diffraction pattern is sampled with $N \geq 3$ spatially separated receiving antennas with the phase centers $\boldsymbol{x}_{a, k}$. Each antenna provides a complex received signal

$E\left(\boldsymbol{x}_{a, k}, t\right)=I\left(\boldsymbol{x}_{a, k}, t\right)+\sqrt{-1} Q\left(\boldsymbol{x}_{a, k}, t\right)$,

where $I$ and $Q$ are the in-phase and quadrature components of the pure return from the scatterers with no noise or clutter, and $k=1,2, \ldots, N$. Without loss of generality, one can consider $\left\langle I\left(\boldsymbol{x}_{a, k}, t\right)\right\rangle=\left\langle Q\left(\boldsymbol{x}_{a, k}, t\right)\right\rangle=0$; hereafter the brackets \langle\rangle denote the ensemble averages. Equations for pure signals can be used directly in practical measurements while noise can be taken into account when calculating SF and CF; see PPa and HDC for details.

\subsection{UCAR-STARS: equations and assumptions}

Consider a pair of receivers with the phase centers $\boldsymbol{x}_{a, k}$ and $\boldsymbol{x}_{a, m},(k \neq m)=1,2, \ldots, N$. The non-dimensional second order cross SF can be defined as (Tatarskii, 1971, chap. 1A):

$D\left(\Delta \boldsymbol{x}_{m k}, \tau\right)=\frac{\left\langle\left[S\left(\boldsymbol{x}_{a, k}, t\right)-S\left(\boldsymbol{x}_{a, k}+\Delta \boldsymbol{x}_{m k}, t+\tau\right)\right]^{2}\right\rangle}{\left\langle\left[S\left(\boldsymbol{x}_{a, k}, t\right)-\left\langle S\left(\boldsymbol{x}_{a, k}, t\right)\right\rangle\right]^{2}\right\rangle}$,

where

$S\left(\boldsymbol{x}_{a, k}, t\right)=E\left(\boldsymbol{x}_{a, k}, t\right) E^{*}\left(\boldsymbol{x}_{a, k}, t\right)$

is the instantaneous power of pure received signals; $\Delta \boldsymbol{x}_{m k}=\boldsymbol{x}_{a, m}-\boldsymbol{x}_{a, k}$ is a spatial separation between the antenna centers; $\tau$ is a temporal separation between the signals, and the superscript ${ }^{*}$ denotes the complex conjugation. The auto SF $D_{\text {auto }}\left(\boldsymbol{x}_{a, k}, \tau\right)$ is a particular case of Eq. (2) at $\Delta \boldsymbol{x}_{m k}=0$. As shown in PPa, the second order SF for any atmospheric profiling radar at $\tau \rightarrow 0$ and small enough $\left|\Delta \boldsymbol{x}_{m k}\right|$ can be presented in the following form:

$$
\begin{aligned}
& D\left(\Delta \boldsymbol{x}_{m k}, \tau\right) \\
& =d_{0}\left(\Delta \boldsymbol{x}_{m k}\right)+d_{1}\left(\Delta \boldsymbol{x}_{m k}\right) \hat{\tau}+d_{2}\left(\Delta \boldsymbol{x}_{m k}\right) \hat{\tau}^{2}+O\left(\tau^{3}\right) \\
& D_{a u t o}\left(\boldsymbol{x}_{a, k}, \tau\right)=d_{a u t o}\left(\boldsymbol{x}_{a, k}\right) \hat{\tau}^{2}+O\left(\tau^{3}\right),
\end{aligned}
$$

where $\hat{\tau}=\tau / \delta t$, and $\delta t$ is the inter-sample time interval. Equations (4) and (5) were derived in PPa using only Assumption 1S: the characteristics of each scatterer $x_{i}(t), y_{i}(t)$, $z_{i}(t), U_{i}(t), V_{i}(t), W_{i}(t)$, and $\Delta n_{i}(t), i=1,2, \ldots, M$, are locally statistically stationary random processes. The term "locally stationary" is used in the paper in the same sense as in a theory of the fine-scale turbulence, e.g. Monin and Yaglom (1975, Sect. 21). It stands for stationarity over a time period which is much smaller than the integral time scale of the random process. Following Assumption 1S, the instantaneous velocity of each scatterer $i=1,2, \ldots, M$ can be presented as a sum of the mean and turbulent components:

$$
\begin{aligned}
& \left\{U_{i}(t), V_{i}(t), W_{i}(t)\right\} \\
& =\left\{\left\langle U_{i}\right\rangle,\left\langle V_{i}\right\rangle,\left\langle W_{i}\right\rangle\right\}+\left\{u_{i}(t), v_{i}(t), w_{i}(t)\right\} .
\end{aligned}
$$

Projection of the instantaneous velocity $\boldsymbol{W}_{i}(t)$ on the baseline $\Delta \boldsymbol{x}_{m k}$ can be defined as follows:

$$
\begin{aligned}
& U_{i, m k}(t) \\
& =\left\langle U_{i, m k}\right\rangle+u_{i, m k}(t)=\left(\boldsymbol{W}_{i}(t) \bullet \Delta \boldsymbol{x}_{m k}\right) /\left|\Delta \boldsymbol{x}_{m k}\right|,
\end{aligned}
$$

where the bullet $\bullet$ denotes a scalar product of two vectors.

To derive practically useful equations for the coefficients $d_{0}$ and $d_{1}$, two more assumptions were adopted in PPa. Assumption $2 \mathrm{~S}$ : the mean motion of all scatterers in the illuminated volume is statistically homogeneous in the horizontal plane $x-y$, that is $\left\langle U_{i}\right\rangle=\langle U\rangle,\left\langle V_{i}\right\rangle=\langle V\rangle$, and $\left\langle U_{i, m k}\right\rangle=\left\langle U_{m k}\right\rangle$ for $i=1,2, \ldots, M$. Assumption 3S: the instantaneous location of all scatterers $x_{i}(t)$ and $y_{i}(t)$ in the volume is statistically uniform in the horizontal plane. Using Assumptions 1S-3S, the following equations were derived in PPa:

$d_{0}\left(\Delta \boldsymbol{x}_{m k}\right)=2\left[1-\exp \left(-\frac{4 \pi^{2} \gamma^{2}\left|\Delta \boldsymbol{x}_{m k}\right|^{2}}{\alpha^{2} D^{2}}\right)\right]$,

$d_{1}\left(\Delta \boldsymbol{x}_{m k}\right)$

$=-\frac{32 \pi^{2} \gamma^{2}\left|\Delta \boldsymbol{x}_{m k}\right|\left\langle U_{m k}\right| \delta t}{\alpha^{2} D^{2}} \exp \left(-\frac{4 \pi^{2} \gamma^{2}\left|\Delta \boldsymbol{x}_{m k}\right|^{2}}{\alpha^{2} D^{2}}\right)$.

Hereafter $D$ is the transmitter diameter; $\alpha^{2}=1+\left(\sigma / \sigma_{a}\right)^{2}$, where $\sigma=\gamma \lambda R / D$ and $\sigma_{a}=\gamma \lambda R / D_{a}$ are the transmitted beam and the receiver's field of view linear widths for a sufficiently large range of observation $R \gg D, D_{a}, \sigma_{r} ; \lambda$ is the radar wavelength; $D_{a}$ is the receiver diameter; $\sigma_{r}$ is the range resolution; and $\gamma$ is the antenna factor. Combining Eqs. (8) and (9), one can obtain:

$\left\langle U_{m k}\right\rangle=\frac{\left|\Delta \boldsymbol{x}_{m k}\right| d_{1}\left(\Delta \boldsymbol{x}_{m k}\right)}{8 \delta t\left[1-d_{0}\left(\Delta \boldsymbol{x}_{m k}\right) / 2\right] \ln \left[1-d_{0}\left(\Delta \boldsymbol{x}_{m k}\right) / 2\right]}$.

This equation relates the projection of the scattering medium's mean speed $\left\langle U_{m k}\right\rangle$ on the baseline $\Delta \boldsymbol{x}_{m k}$ to "measurable" coefficients $d_{0}$ and $d_{1}$ in Eq. (4) for cross SF. The mean horizontal wind speed components $\langle U\rangle$ and $\langle V\rangle$ can be estimated uniquely by applying Eq. (10) to the coefficients $d_{0}\left(\Delta \boldsymbol{x}_{m k}\right)$ and $d_{1}\left(\Delta \boldsymbol{x}_{m k}\right)$ for any two non-parallel baselines $\Delta \boldsymbol{x}_{m k}$ at $(m \neq k)=1,2, \ldots, N$. To derive practically useful equations for coefficients $d_{2}$ and $d_{\text {auto }}$, the following additional assumptions were adopted in $\mathrm{PPa}$ and $\mathrm{PPb}$. Assumption 4S: turbulent motion of all scatterers inside the illuminated volume is statistically homogeneous, that is $\left\langle w_{i}^{p}\right\rangle=\left\langle w^{p}\right\rangle,\left\langle u_{i, m k}^{p}\right\rangle=\left\langle u_{m k}^{p}\right\rangle,\left\langle u_{i}^{j} v_{i}^{p-j}\right\rangle=\left\langle u^{j} v^{p-j}\right\rangle$ for $i=1,2, \ldots, M$, and $j=0,1, \ldots, p$. Assumption 5S: the integral scale of the vertical turbulent velocity $w_{i}(t)$ is smaller than $\sigma_{r}$ and/or $\sigma$, and that of the horizontal velocities $u_{i}(t)$ and $v_{i}(t)$ is approximately equal to, or larger than, $\sigma$ for $i=1,2, \ldots, M$. Using Assumptions 1S-5S, the following equations were derived in $\mathrm{PPa}$ and $\mathrm{PPb}$ : 
$d_{2}\left(\Delta \boldsymbol{x}_{m k}\right)=32 \pi^{2} \delta t^{2}\left[\frac{\left\langle w^{2}\right\rangle}{\lambda^{2}}+\frac{\gamma^{2}\left(\langle U\rangle^{2}+\langle V\rangle^{2}\right)}{\alpha^{2} D^{2}}-\frac{8 \pi^{2} \gamma^{4}\left|\Delta \boldsymbol{x}_{m k}\right|^{2}\left(\left|U_{m k}\right\rangle^{2}+\left\langle u_{m k}^{2}\right\rangle\right)}{\alpha^{4} D^{4}}\right] \exp \left(-\frac{4 \pi^{2} \gamma^{2}\left|\Delta \boldsymbol{x}_{m k}\right|^{2}}{\alpha^{2} D^{2}}\right)$

$d_{\text {auto }}\left(\boldsymbol{x}_{a, k}\right)=32 \pi^{2} \delta t^{2}\left[\frac{\left\langle w^{2}\right\rangle}{\lambda^{2}}+\frac{\gamma^{2}\left(\langle U\rangle^{2}+\langle V\rangle^{2}\right)}{\alpha^{2} D^{2}}\right]$.

Equation (12) relates the variance of the vertical turbulent velocity $\left\langle w^{2}\right\rangle$ to the "measurable" coefficient $d_{\text {auto }}$ in Eq. (5) for the auto SF. Combining Eqs. (8), (11), and (12) with the standard expression for the instantaneous value $U_{m k}(t)$ for a baseline $\Delta \boldsymbol{x}_{m k}=\left\{\Delta x_{m k}, \Delta y_{m k}, 0\right\}$ (Doviak and Zrnić, 1993, Sect. 9.3), the following relation was derived in PPb:

$$
\begin{aligned}
& \left(\left\langle u^{2}\right\rangle+\langle U\rangle^{2}\right) \Delta x_{m k}^{2}+2(\langle u v\rangle+\langle U\rangle\langle V\rangle) \Delta x_{m k} \Delta y_{m k}+\left(\left\langle v^{2}\right\rangle+\langle V\rangle^{2}\right) \Delta y_{m k}^{2} \\
& =\frac{\left(\Delta x_{m k}^{2}+\Delta y_{m k}^{2}\right)^{2}}{16 \ln ^{2}\left[1-d_{0}\left(\Delta \boldsymbol{x}_{m k}\right) / 2\right] \delta t^{2}}\left[d_{\text {auto }}\left(\boldsymbol{x}_{a, k}\right)-\frac{d_{2}\left(\Delta \boldsymbol{x}_{m k}\right)}{1-d_{0}\left(\Delta \boldsymbol{x}_{m k}\right) / 2}\right] .
\end{aligned}
$$

This linear equation relates three unknown values, the turbulence characteristics $\left\langle u^{2}\right\rangle,\left\langle v^{2}\right\rangle$, and $\langle u v\rangle$ to "measurable" coefficients $d_{0}, d_{2}$, and $d_{\text {auto }}$ in Eqs. (4) and (5) for the secondorder cross and auto SF. The characteristics $\left\langle u^{2}\right\rangle,\left\langle v^{2}\right\rangle$, and $\langle u v\rangle$ can be estimated uniquely by applying Eq. (13) to the coefficients $d_{0}\left(\Delta \boldsymbol{x}_{m k}\right), d_{2}\left(\Delta \boldsymbol{x}_{m k}\right)$, and $d_{a u t o}\left(\boldsymbol{x}_{a, k}\right)$ for any three non-parallel baselines $\Delta \boldsymbol{x}_{m k}$ at $(m \neq k)=1,2, \ldots, N$.

Equations (2)-(5), (10), (12), and (13) are the major operational equations for measuring the mean horizontal winds and the second-order turbulence characteristics with the UCAR-STARS method.

\subsection{HAD: equations and assumptions}

The non-dimensional second order cross CF for a pair of receivers with the phase centers $\boldsymbol{x}_{a, k}$ and $\boldsymbol{x}_{a, m}$, $(k \neq m)=1,2, \ldots, N$, can be defined as:

$$
C\left(\Delta \boldsymbol{x}_{m k}, \tau\right)=\frac{\left\langle E\left(\boldsymbol{x}_{a, k}, t\right) E^{*}\left(\boldsymbol{x}_{a, k}+\Delta \boldsymbol{x}_{m k}, t+\tau\right)\right\rangle}{\left\langle E\left(\boldsymbol{x}_{a, k}\right) E^{*}\left(\boldsymbol{x}_{a, k}\right)\right\rangle} .
$$

The auto CF $C_{a u t o}\left(\boldsymbol{x}_{a, k}, \tau\right)$ is a particular case of Eq. (14) at $\Delta \boldsymbol{x}_{m k}=0$. Equations (1)-(6) in HDC for the magnitude of cross and auto CF can be presented in the following form that matches notations in Sect. 2.1:

$$
\begin{aligned}
& \left|C\left(\Delta \boldsymbol{x}_{m k}, \tau\right)\right| \\
& =\exp \left[-c_{0}\left(\Delta \boldsymbol{x}_{m k}\right)-c_{1}\left(\Delta \boldsymbol{x}_{m k}\right) \hat{\tau}-c_{2}\left(\Delta \boldsymbol{x}_{m k}\right) \hat{\tau}^{2}-O\left(\tau^{3}\right)\right] \\
& \left|C_{\text {auto }}\left(\boldsymbol{x}_{a, k}, \tau\right)\right|=\exp \left[-c_{\text {auto }}\left(\boldsymbol{x}_{a, k}\right) \hat{\tau}^{2}-O\left(\tau^{3}\right)\right],
\end{aligned}
$$

where the coefficients are given as follows:

$$
\begin{aligned}
& c_{0}\left(\Delta \boldsymbol{x}_{m k}\right)=\frac{2 \pi^{2} \gamma^{2}\left|\Delta \boldsymbol{x}_{m k}\right|^{2}}{\alpha^{2} D^{2}} \\
& c_{1}\left(\Delta \boldsymbol{x}_{m k}\right)=-\frac{8 \pi^{2} \gamma^{2}\left|\Delta \boldsymbol{x}_{m k}\right|\left|U_{m k}\right\rangle \delta t}{\alpha^{2} D^{2}} \\
& c_{2}\left(\Delta \boldsymbol{x}_{m k}\right)=c_{\text {auto }}\left(\boldsymbol{x}_{a, k}\right) \\
& c_{\text {auto }}\left(\boldsymbol{x}_{a, k}\right)=8 \pi^{2} \delta t^{2}\left[\frac{\left\langle w^{2}\right\rangle}{\lambda^{2}}+\frac{\gamma^{2}\left(\langle U\rangle^{2}+\langle V\rangle^{2}\right)}{\alpha^{2} D^{2}}\right] .
\end{aligned}
$$

Combining Eqs. (17) and (18), one can obtain:

$\left\langle U_{m k}\right\rangle=-\frac{\left|\Delta \boldsymbol{x}_{m k}\right|}{4 \delta t} \frac{c_{1}\left(\Delta \boldsymbol{x}_{m k}\right)}{c_{0}\left(\Delta \boldsymbol{x}_{m k}\right)}$.

Equations (19)-(21) relate the mean speed $\left\langle U_{m k}\right\rangle$ and variance of the vertical turbulent velocity $\left\langle w^{2}\right\rangle$ to "measurable" coefficients $c_{0}, c_{1}, c_{2}$, and $c_{\text {auto }}$ in Eqs. (15) and (16) for the second-order cross and auto CF. The characteristics $\langle U\rangle,\langle V\rangle$, and $\left\langle w^{2}\right\rangle$ can be estimated uniquely by applying Eqs. (14)-(16) and Eqs. (19)-(21) to any two non-parallel baselines $\Delta \boldsymbol{x}_{m k}$ at $(m \neq k)=1,2, \ldots, N$.

Equations (14)-(16) and Eqs. (19)-(21) are the major operational equations for measuring the mean horizontal winds and variance of the vertical turbulent velocity with the HAD method. The assumptions which were adopted for deriving Eqs. (15)-(20) are not listed systematically in DLH but rather scattered throughout the paper. Below we systemize the assumptions, and present them in the terms of the notation adopted in Sect. 2.1 whenever possible:

- Assumption 1H: the characteristics of each scatterer $x_{i}(t), y_{i}(t), z_{i}(t), U_{i}(t), V_{i}(t), W_{i}(t)$, and $\Delta n_{i}(t)$, $i=1,2, \ldots, M$, are globally statistically stationary random processes (DLH, p. 161).

- Assumption 2H: the instantaneous location of all scatterers is statistically uniform in the illuminated volume (DLH, pp. 158 and 161).

- Assumption 3H: the mean motion of all scatterers is statistically homogeneous in the illuminated volume; that is $\left\langle U_{i}\right\rangle=\langle U\rangle,\left\langle V_{i}\right\rangle=\langle V\rangle$, and $\left\langle W_{i}\right\rangle=\langle W\rangle$ (DLH, p. 163).

- Assumption 4H: the instantaneous reflectivity of all scatterers is statistically homogeneous in the illuminated volume (DLH, pp. 158 and 161).

- Assumption 5H: turbulent motion of all scatterers inside the illuminated volume is statistically homogeneous and isotropic (DLH, p. 163 and Sect. 4). Following 
this assumption, turbulence was characterized in DLH DHL (Sect. 5.3), $\sigma_{t}$ is related to the spectral width in the Doppler method, therefore, the measured value in Eq. (19) is $\left\langle w^{2}\right\rangle$.

- Assumption 6H: specific functional form of CF or spectrum for the reflectivity $\Delta n_{i}$ of scatterers in the illuminated volume; e.g. the Gaussian $\mathrm{CF}$ with the correlation lengths $\rho_{c h}$ and $\rho_{c z}$ in the horizontal and vertical directions (DLH, Sect. 4), a power law of the Kolmogorov type with specified parameters (DLH, Sect. 5), or other.

- Assumption 7H: the vertical correlation length $\rho_{c z}$ is much smaller than the range resolution $\sigma_{r}$ (DLH, Sect. 4.4).

- Assumption 8H: specific horizontal correlation length $\rho_{c h}$; e.g. $\rho_{c h} \ll D$ (DHL, pp. 159, 166), $\rho_{c h} \geq D$, $\rho_{c h} \gg \rho_{c z}$ (DLH, p. 170), or other. and HDC by $\sigma_{t}=\sqrt{\left\langle w^{2}\right\rangle}=\sqrt{\left\langle u^{2}\right\rangle}=\sqrt{\left\langle v^{2}\right\rangle}$. As shown in

\subsection{Relations between STARS and HAD}

To relate the second order $\mathrm{CF}$ and $\mathrm{SF}$, one can apply the standard set of assumptions about the received signals $E\left(\boldsymbol{x}_{a, k}, t\right)$; the assumptions are presented and discussed by Ishimaru (1997, Sect. 4-9). The complex received signal in Eq. (1) can also be presented as $E\left(\boldsymbol{x}_{a, k}, t\right)=A\left(\boldsymbol{x}_{a, k}, t\right) \exp \left[\sqrt{-1} \phi\left(\boldsymbol{x}_{a, k}, t\right)\right]$, where $A$ and $\phi$ are the amplitude and the phase of the signal, and $k=1,2, \ldots, N$. Let us consider the in-phase and the quadrature components $I\left(\boldsymbol{x}_{a, k}, t\right)$ and $Q\left(\boldsymbol{x}_{a, k}, t\right)$ in Eq. (1) as two statistically stationary and independent Gaussian random processes, and the phase $\phi$ to be uniformly distributed over $2 \pi$. Let us further consider the joint distribution of $I\left(\boldsymbol{x}_{a, k}, t\right), Q\left(\boldsymbol{x}_{a, k}, t\right), I\left(\boldsymbol{x}_{a, k}+\Delta \boldsymbol{x}_{m k}, t+\tau\right)$, and $Q\left(\boldsymbol{x}_{a, k}+\Delta \boldsymbol{x}_{m k}, t+\tau\right)$ to be Gaussian as well, and the antenna centers to be close to each other. The relevant consequences from these assumptions can be reproduced from Ishimaru (1997, Sect. 4-9) in our notations as follows:

$$
\begin{aligned}
& \left\langle S\left(\boldsymbol{x}_{a, k}, t\right)\right\rangle=\left\langle S\left(\boldsymbol{x}_{a, k}+\Delta \boldsymbol{x}_{m k}, t+\tau\right)\right\rangle=\langle S\rangle \\
& \left\langle E\left(\boldsymbol{x}_{a, k}, t\right) E\left(\boldsymbol{x}_{a, k}\right), t\right\rangle \\
& \approx\left\langle E\left(\boldsymbol{x}_{a, k}, t\right) E\left(\boldsymbol{x}_{a, k}+\Delta \boldsymbol{x}_{m k}, t+\tau\right)\right\rangle \approx 0 .
\end{aligned}
$$

Applying Eq. (22) to Eq. (2), one can present cross SF as follows:

$D\left(\Delta \boldsymbol{x}_{m k}, \tau\right)=\frac{\left\langle S^{2}\left(\boldsymbol{x}_{a, k}, t\right)\right\rangle-2\left\langle S\left(\boldsymbol{x}_{a, k}, t\right) S\left(\boldsymbol{x}_{a, k}+\Delta \boldsymbol{x}_{m k}, t+\tau\right)\right\rangle+\left\langle S^{2}\left(\boldsymbol{x}_{a, k}+\Delta \boldsymbol{x}_{m k}, t+\tau\right)\right\rangle}{\left\langle S^{2}\left(\boldsymbol{x}_{a, k}, t\right)\right\rangle-\langle S\rangle^{2}}$.

Using Eq. (3), the terms $\left\langle S^{2}\left(\boldsymbol{x}_{a, k}, t\right)\right\rangle,\left\langle S\left(\boldsymbol{x}_{a, k}, t\right) S\left(\boldsymbol{x}_{a, k}+\Delta \boldsymbol{x}_{m k}, t+\tau\right)\right\rangle$, and $\left\langle S^{2}\left(\boldsymbol{x}_{a, k}+\Delta \boldsymbol{x}_{m k}, t+\tau\right)\right\rangle$ can be presented as the fourth moments. For example,

$\left\langle S\left(\boldsymbol{x}_{a, k}, t\right) S\left(\boldsymbol{x}_{a, k}+\Delta \boldsymbol{x}_{m k}, t+\tau\right)\right\rangle=\left\langle E\left(\boldsymbol{x}_{a, k}, t\right) E^{*}\left(\boldsymbol{x}_{a, k}, t\right) E\left(\boldsymbol{x}_{a, k}+\Delta \boldsymbol{x}_{m k}, t+\tau\right) E^{*}\left(\boldsymbol{x}_{a, k}+\Delta \boldsymbol{x}_{m k}, t+\tau\right)\right\rangle$.

The in-phase and the quadrature components are considered Gaussian with zero mean, therefore, the fourth moment of the complex signal can be expressed in terms of the products of the second moments as (e.g. Doviak and Zrnić, 1993, Sect. 5.1):

$\left\langle E\left(\boldsymbol{x}_{a, k}, t\right) E^{*}\left(\boldsymbol{x}_{a, k}, t\right) E\left(\boldsymbol{x}_{a, k}+\Delta \boldsymbol{x}_{m k}, t+\tau\right) E^{*}\left(\boldsymbol{x}_{a, k}+\Delta \boldsymbol{x}_{m k}, t+\tau\right)\right\rangle$

$=\left\langle E\left(\boldsymbol{x}_{a, k}, t\right) E^{*}\left(\boldsymbol{x}_{a, k}, t\right)\right\rangle\left\langle E\left(\boldsymbol{x}_{a, k}+\Delta \boldsymbol{x}_{m k}, t+\tau\right) E^{*}\left(\boldsymbol{x}_{a, k}+\Delta \boldsymbol{x}_{m k}, t+\tau\right)\right\rangle$

$+\left\langle E\left(\boldsymbol{x}_{a, k}, t\right) E\left(\boldsymbol{x}_{a, k}+\Delta \boldsymbol{x}_{m k}, t+\tau\right)\right\rangle\left\langle E^{*}\left(\boldsymbol{x}_{a, k}, t\right) E^{*}\left(\boldsymbol{x}_{a, k}+\Delta \boldsymbol{x}_{m k}, t+\tau\right)\right\rangle$

$+\left\langle E\left(\boldsymbol{x}_{a, k}, t\right) E^{*}\left(\boldsymbol{x}_{a, k}+\Delta \boldsymbol{x}_{m k}, t+\tau\right)\right\rangle\left\langle E^{*}\left(\boldsymbol{x}_{a, k}, t\right) E\left(\boldsymbol{x}_{a, k}+\Delta \boldsymbol{x}_{m k}, t+\tau\right)\right\rangle$.

Using Eqs. (14), (22), and (23), the RHS of Eq. (25) can be reduced to the compact form:

$\langle S\rangle^{2}+\langle S\rangle^{2} C\left(\Delta \boldsymbol{x}_{m k}, \tau\right) C^{*}\left(\Delta \boldsymbol{x}_{m k}, \tau\right)=\langle S\rangle^{2}\left[1+\left|C\left(\Delta \boldsymbol{x}_{m k}, \tau\right)\right|^{2}\right]$.

Similarly one can obtain $\left\langle S^{2}\left(\boldsymbol{x}_{a, k}, t\right)\right\rangle=\left\langle S^{2}\left(\boldsymbol{x}_{a, k}+\Delta \boldsymbol{x}_{m k}, t+\tau\right)\right\rangle=2\langle S\rangle^{2}$, and present Eq. (24) as follows:

$D\left(\Delta \boldsymbol{x}_{m k}, \tau\right)=2\left[1-\left|C\left(\Delta \boldsymbol{x}_{m k}, \tau\right)\right|^{2}\right]$

This equation relates the second order CF and SF for received signals and provides a "bridge" between SF and CF-based SA techniques. In particular, one can establish direct relations between HAD and STARS with Eq. (27).

It follows from Eqs. (4) and (5) that:

$$
\begin{array}{ll}
d_{0}\left(\Delta \boldsymbol{x}_{m k}\right)=D\left(\Delta \boldsymbol{x}_{m k}, 0\right), & d_{1}\left(\Delta \boldsymbol{x}_{m k}\right)=\left[\frac{\partial D\left(\Delta \boldsymbol{x}_{m k}, \tau\right)}{\partial \tau}\right]_{\tau=0}, \\
d_{2}\left(\Delta \boldsymbol{x}_{m k}\right)=\frac{1}{2}\left[\frac{\partial^{2} D\left(\Delta \boldsymbol{x}_{m k}, \tau\right)}{\partial \tau^{2}}\right]_{\tau=0}, & d_{\text {auto }}\left(\boldsymbol{x}_{a, k}\right)=\frac{1}{2}\left[\frac{\partial^{2} D_{a u t o}\left(\boldsymbol{x}_{a, k}, \tau\right)}{\partial \tau^{2}}\right]_{\tau=0} .
\end{array}
$$


Using Eqs. (27), (28), (15), and (16), one can estimate coefficients $d_{0}, d_{1}, d_{2}$, and $d_{\text {auto }}$ in Eqs. (4) and (5) using the cross and auto $\mathrm{CF}$ as follows (the estimates are denoted by the tilde):

$$
\begin{aligned}
\tilde{d}_{0}\left(\Delta \boldsymbol{x}_{m k}\right) & =2\left[1-\left|C\left(\Delta \boldsymbol{x}_{m k}, 0\right)\right|^{2}\right]=2\left\{1-\exp \left[-2 c_{0}\left(\Delta \boldsymbol{x}_{m k}\right)\right]\right\}=d_{0}\left(\Delta \boldsymbol{x}_{m k}\right) \\
\tilde{d}_{1}\left(\Delta \boldsymbol{x}_{m k}\right) & =-2\left[\frac{\partial\left|C\left(\Delta \boldsymbol{x}_{m k}, \tau\right)\right|^{2}}{\partial \tau}\right]_{\tau=0}=4 c_{1}\left(\Delta \boldsymbol{x}_{m k}\right) \exp \left[-2 c_{0}\left(\Delta \boldsymbol{x}_{m k}\right)\right]=d_{1}\left(\Delta \boldsymbol{x}_{m k}\right) \\
\tilde{d}_{2}\left(\Delta \boldsymbol{x}_{m k}\right) & =-\left[\frac{\partial^{2}\left|C\left(\Delta \boldsymbol{x}_{m k}, \tau\right)\right|^{2}}{\partial \tau^{2}}\right]_{\tau=0}=4\left[c_{2}\left(\Delta \boldsymbol{x}_{m k}\right)-c_{1}^{2}\left(\Delta \boldsymbol{x}_{m k}\right)\right] \exp \left[-2 c_{0}\left(\Delta \boldsymbol{x}_{m k}\right)\right] \\
& =d_{2}\left(\Delta \boldsymbol{x}_{m k}\right)+\frac{256 \pi^{4} \gamma^{4}\left|\Delta \boldsymbol{x}_{m k}\right|^{2} \delta t^{2}\left\langle u_{m k}^{2}\right\rangle}{\alpha^{4} D^{4}} \exp \left(-\frac{4 \pi^{2} \gamma^{2}\left|\Delta \boldsymbol{x}_{m k}\right|^{2}}{\alpha^{2} D^{2}}\right) \\
\tilde{d}_{\text {auto }}\left(\boldsymbol{x}_{a, k}\right) & =-\left[\frac{\partial^{2}\left|C_{a u t o}\left(\boldsymbol{x}_{a, k}, \tau\right)\right|^{2}}{\partial \tau^{2}}\right]_{\tau=0}=4 c_{\text {auto }}\left(\boldsymbol{x}_{a, k}\right)=d_{\text {auto }}\left(\boldsymbol{x}_{a, k}\right),
\end{aligned}
$$

where $d_{0}, d_{1}, d_{2}$, and $d_{\text {auto }}$ are given by Eqs. (8), (9), (11), and (12). One can see that coefficients $d_{2}\left(\Delta \boldsymbol{x}_{m k}\right)$ and $\tilde{d}_{2}\left(\Delta \boldsymbol{x}_{m k}\right)$ differ by the term with variance of the horizontal turbulent velocity $\left\langle u_{m k}^{2}\right\rangle$.

\section{Experimental results}

The Middle and Upper atmosphere Radar (MUR) is located in Shigaraki, Japan $\left(34.85^{\circ} \mathrm{N}, 136.10^{\circ} \mathrm{E}\right)$ at approximately $1 \mathrm{~km}$ above sea level. The radar is operated by the Radio Science Center for Space and Atmosphere of Kyoto University. MUR is the VHF band MST Doppler research radar with the operational frequency $46.5 \mathrm{MHz}$ and the corresponding wavelength $\lambda=6.45 \mathrm{~m}$. It is an active phased array system; the radar's antenna consists of 475 crossed Yagis with the aperture $8330 \mathrm{~m}^{2}$ (103 $\mathrm{m}$ in diameter). A detailed description of the MU radar can be found in Fukao et al. (1985a, b).

Starting in November 1984, MUR was successfully used for studying various dynamic processes in the turbosphere, stratosphere-troposphere interaction, and many other phenomena at altitudes ranging from $5 \mathrm{~km}$ to $100 \mathrm{~km}$. Being equipped with very flexible radar control and data acquisition systems, MUR was intensively used for testing and improvement of new data analysis techniques (e.g. Tsuda et al., 1985; Van Baelen et al., 1990; Palmer et al., 1990a, b and 1993; Chilson et al., 1992; Sheppard et al., 1993; Luce et al., 2000; and Hysell et al., 2002). The flexible radar control and data acquisition systems made the MUR a natural test bed for evaluating the UCAR-STARS method.

\subsection{Experimental setup}

The data collection for testing UCAR-STARS was executed on 24-25 April 2002. Because MUR can be operated both in SA and DBS modes, the experiments were designed in the following way. The radar was operated $6.55 \mathrm{~min}$ in the SA mode, then approximately one minute in the DBS mode; the sequence was continuously repeated for several hours for each studied configuration of receiving antennas.
A full MUR antenna is separated into 25 groups with 19 Yagis in each group; Fig. 1. Any group or groups can be used either separately, or in arbitrary combinations for both transmitting and receiving; this feature ensures MUR's unique flexibility. Both in DBS and SA modes, a full antenna (475 Yagis) was transmitting at the pulse repetition frequency $2500 \mathrm{~Hz}$. The range bin and the gate separation were $150 \mathrm{~m}$; 64 gates covered a height $z_{o}$ from $5 \mathrm{~km}$ to $15 \mathrm{~km}$ above the radar. The MUR transmitted beam width ( $-3 \mathrm{~dB}$ points) for a full antenna is $3.6^{\circ}$.

In DBS mode, pulses were transmitted sequentially in five directions (vertical, N, E, S, W) using the full antenna array, with $10^{\circ}$ tilt from zenith for the non-vertical beams. The full antenna array was used to receive signals. The number of coherent integrations (NCI) was 38 , the number of incoherent integrations was 6 , and the number of FFT points was 128 , hence the averaging time was $T_{a v}=58.4 \mathrm{~s}$. The standard DBS processing for the MUR was applied, by removing DC bias in the time-series data from each receiver channel before FFT calculation, and then carrying out Gaussian fitting around the spectral peak in the integrated data to obtain the Doppler parameters: reflectivity, radial velocity, and spectral width.

There were four receiving antennas in the SA mode. The NCI was 128, the length of one record was 256 samples, and 30 records covering $6.55 \mathrm{~min}$ were recorded continuously between switches to the DBS mode. Three different configurations of receivers were studied in the SA mode. The results for all configurations are practically identical, and only those for a configuration in Fig. 1 are presented in this paper. One can see in Fig. 1 that each receiver consists of 7 groups (133 Yagis) with a diameter of approximately $54 \mathrm{~m}$. The receiver centers provide six antenna-pairs (baselines), and no two baselines are parallel to one other.

\subsection{Measurements of mean winds}

The mean horizontal wind speed components $\langle U\rangle$ (towards east) and $\langle V\rangle$ (towards north) were retrieved from the MUR signals in the SA mode with STARS and HAD techniques at 

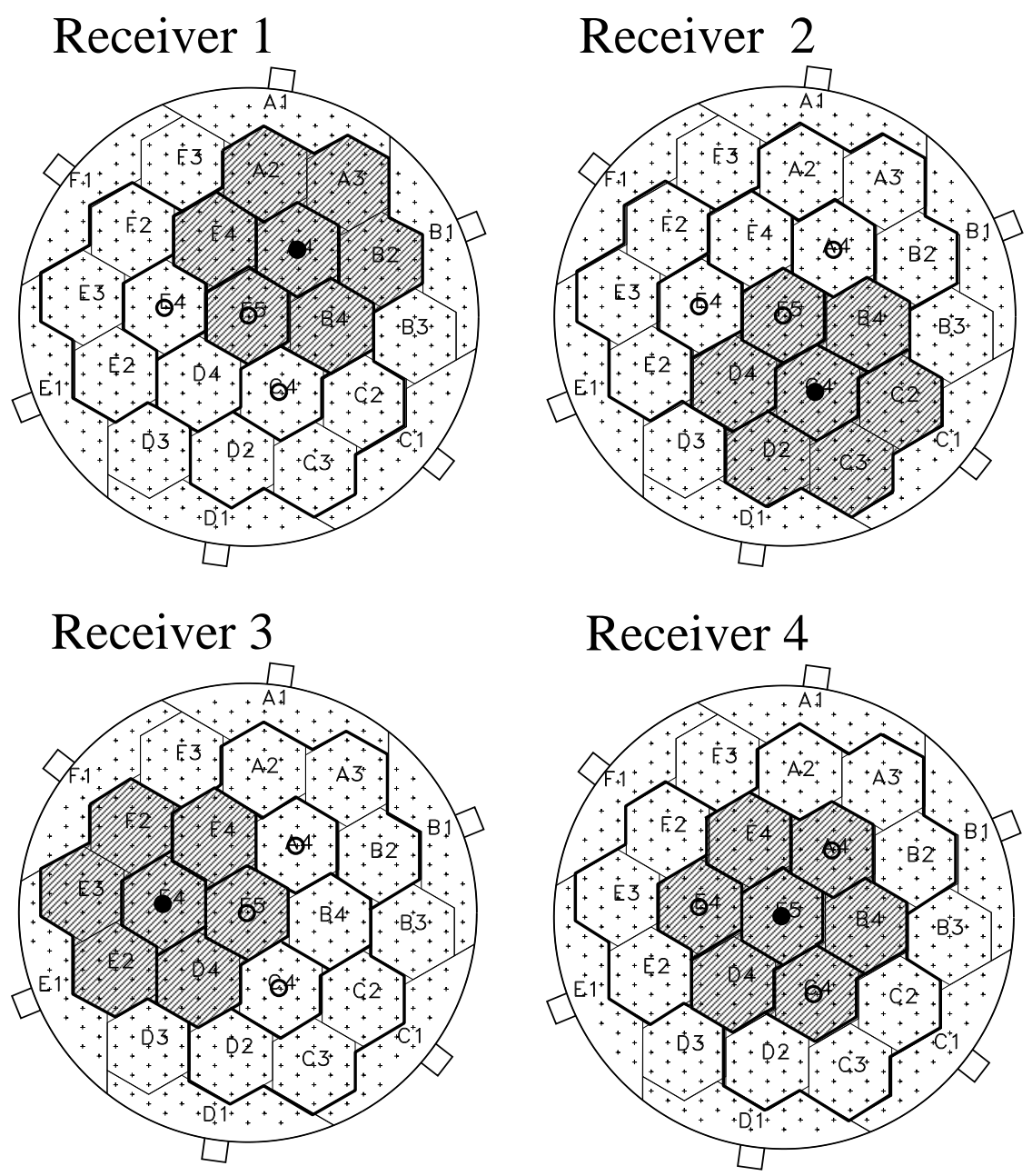

Fig. 1. A schematic depiction of $N=4$ receiving antennas for the MUR experiment on 24 April 2002. Each receiver is shown by shading and consists of 7 groups, 19 Yagis per group. In each drawing, the receiver's center is indicated by a bullet while circles show centers of the other three receivers.

the same averaging time $T_{a v}=78.6 \mathrm{~s}$ which is close enough to the DBS $T_{a v}=58.4 \mathrm{~s}$. Typical vertical profiles of $\langle U\rangle,\langle V\rangle$, and SNR are presented in Fig. 2. One can note a difference between SNR estimates by different methods in this and the following figures. SNR is shown as it was produced by the methods without correcting for difference in NCI and size of receiving antennas in SA and DBS modes. Furthermore, specific techniques for estimating noise in DBS, HAD, and STARS methods are different. The difference in measured values of SNR is natural, and the values themselves are shown only for illustrating a trend in the SNR with a height.

Very rough data rejection criterion for the outliers in the DBS-measured mean wind speed components was applied. The average and RMS values for $\langle U\rangle$ and $\langle V\rangle$ were estimated over the total 2.5-h experimental period for each gate separately. The DBS-measured wind speed component was then rejected when it differed from the average by more than 6 RMS values for the gate. The STARS-measured values of $\langle U\rangle$ and $\langle V\rangle$ were estimated when SNR for actual received signals over a 78.6-s interval was equal to or greater than $-21.2 \mathrm{~dB}$. The HAD estimates of $\langle U\rangle$ and $\langle V\rangle$ were calculated when the SNR of the received signals over a 78.6-s interval was equal to or greater than $-30.0 \mathrm{~dB}$, the spectra of the signals did not contain detectable interference, the standard deviation of auto- and cross-correlation function widths divided by their mean width was equal to or less than 0.2 , and the noise-corrected cross-correlation coefficients at zero time-lag were equal to or greater than 0.1.

Figure 2 illustrates a well-known feature of the atmospheric flow above the MUR: a stable jet directed practically due east; e.g. Van Baelen et al. (1990), Hassenpflug et al. (2003). The jet consists of two clearly distinguishable parts: the low-speed part below $z_{o} \approx 10 \mathrm{~km}$, and the highspeed part at $z_{o} \approx 11-14 \mathrm{~km}$. One can see that SNR is rather high below $z_{o} \approx 11 \mathrm{~km}$ while it decreases significantly in the high-speed flow. The profiles of $\langle U\rangle$ and $\langle V\rangle$ demonstrate reasonable agreement of SA and DBS results at lower altitudes, and much poorer agreement above $z_{o} \approx 11 \mathrm{~km}$. One can also notice a rather significant value of the northerly component 

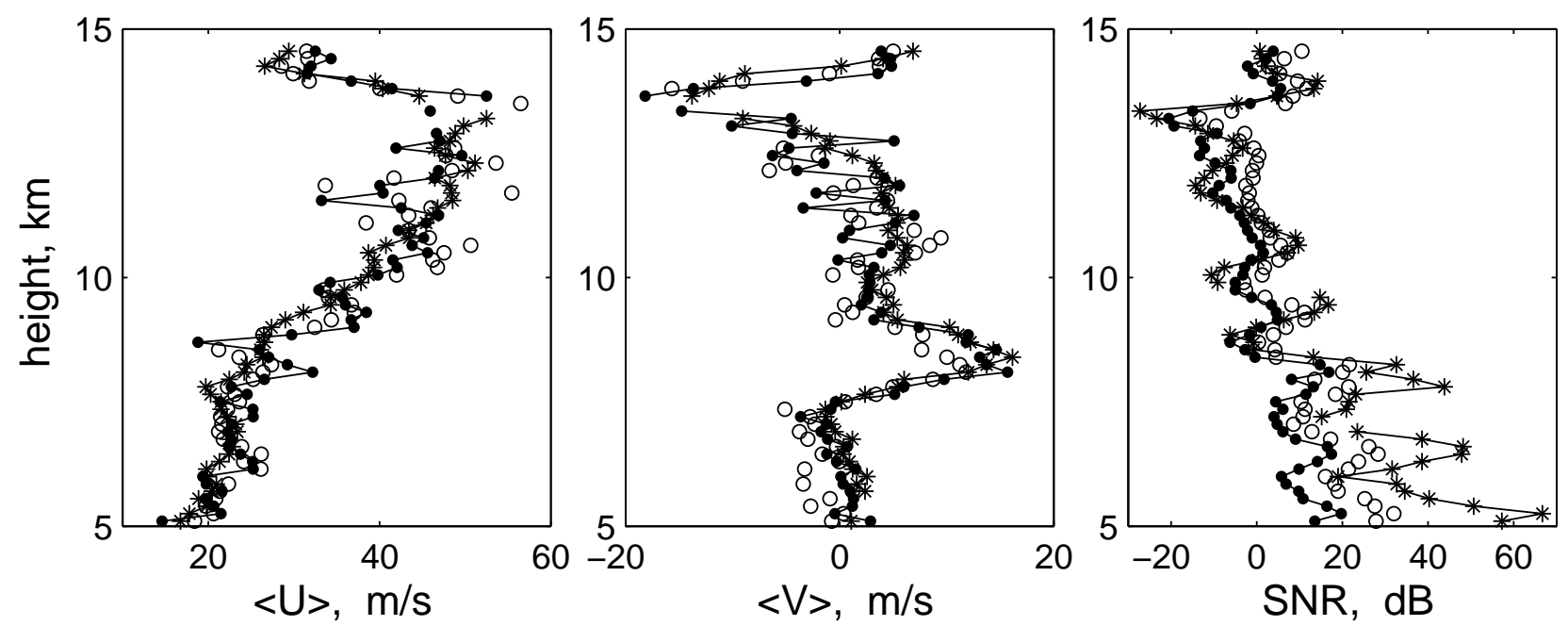

Fig. 2. The vertical profiles of the easterly $\langle U\rangle$ and northerly $\langle V\rangle$ mean horizontal wind speed components and SNR above the MUR on 24 April 2002 at 21:51:26 LT. •, STARS; o, HAD; *, DBS.
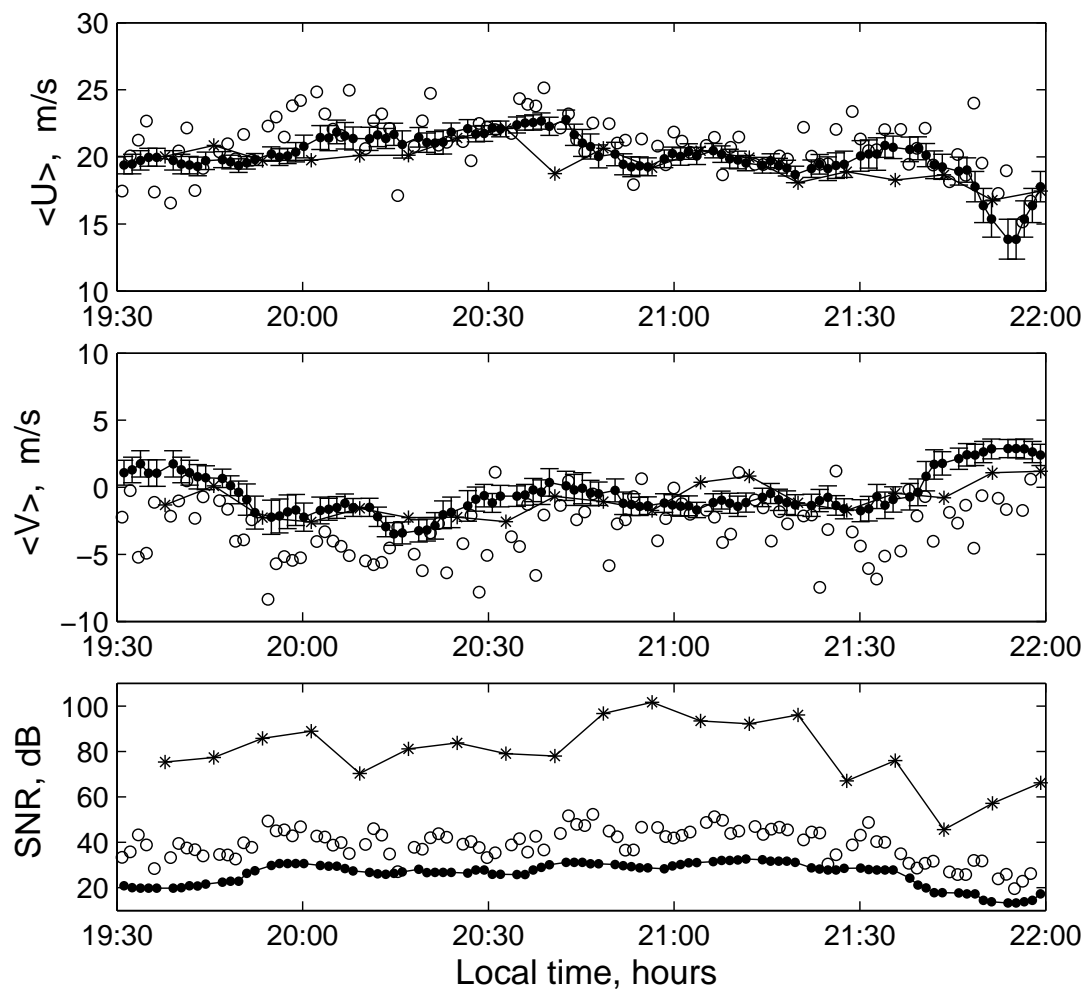

Fig. 3. Time series of the easterly $\langle U\rangle$ and northerly $\langle V\rangle$ mean wind speed components and SNR on 24 April 2002 at $z_{o}=5.1 \mathrm{~km}$. •, STARS; $\circ, \mathrm{HAD} ; *$, DBS.

$\langle V\rangle$ around $z_{o} \approx 8 \mathrm{~km}$, which indicates a change in the jet direction from pure east to the north-east at these heights. To evaluate the potential applicability of STARS to measuring mean winds with the MUR, only results at $z_{o}=5-11 \mathrm{~km}$ are considered below.
Typical time series of the mean horizontal winds and SNR in the low-speed part of the jet are shown in Figs. 3 and 4. Experimental error bars are shown for the STARS estimates of $\langle U\rangle$ and $\langle V\rangle$ in Figs. 3, 4, and errors for turbulence characteristics are shown in Figs. 8, 9. The errors were estimated in the following way. As shown in Sect. 2.1, any two 

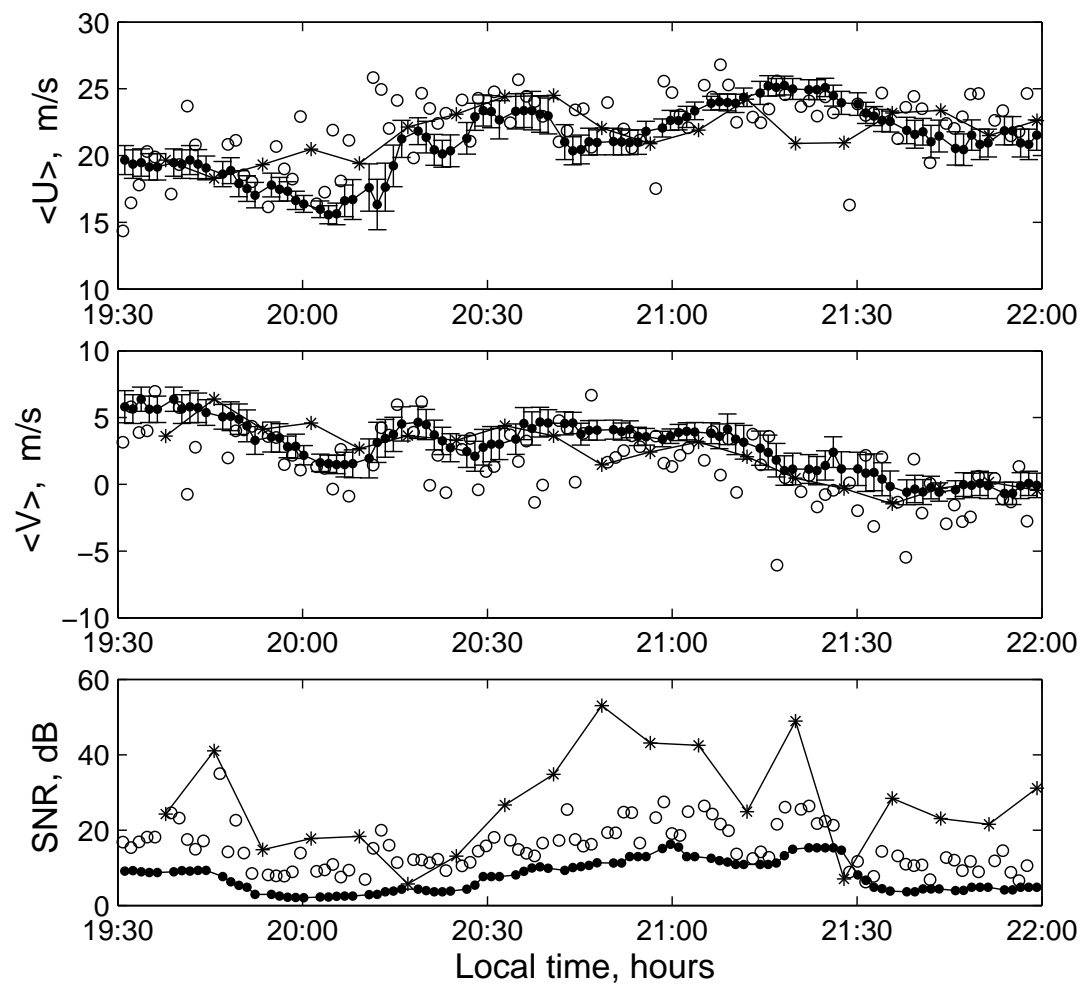

Fig. 4. The same as in Fig. 3 at $z_{o}=7.5 \mathrm{~km}$.

non-parallel baselines provide a unique estimate for $\langle U\rangle$ and $\langle V\rangle$ with Eq. (10). Therefore, any three non-parallel baselines provide three estimates for $\langle U\rangle$ and $\langle V\rangle$ (three pairs in a triangle). Four actual signals from four MUR receivers in Fig. 1 allow one to employ two fully independent triangles: baselines $(1,2),(2,3),(1,3)$; and $(1,4),(2,4),(3,4)$. The triangles provide 6 estimates for $\langle U\rangle$ and $\langle V\rangle$ for each 78.6$\mathrm{s}$ interval. One can also simulate the combined receiving antennas by combining the actual signals. For example, a signal $E\left(\boldsymbol{x}_{a, 1}, t\right)+E\left(\boldsymbol{x}_{a, 2}, t\right)$ is that from the 12-group receiving antenna (Receivers 1 and 2 in Fig. 1) with a center $\left(\boldsymbol{x}_{a, 1}+\boldsymbol{x}_{a, 2}\right) / 2$. One can further simulate combined receivers by using different sums of three actual signals. SF is defined by Eq. (2) for the instantaneous signal power which is a nonlinear combination $I^{2}+Q^{2}$ of the $I$ and $Q$ components, Eq. (3). Therefore, the combined signals contain different information from that in the actual signals. Although one can obtain a very large number of estimates this way, only a small fraction of them are independent. We were able to define seven independent triangles from both actual and combined signals for a configuration in Fig. 1 which produced 21 estimates for $\langle U\rangle$ and $\langle V\rangle$ for each 78.6-s interval. Theoretically, all the estimates should be identical, although it is never the case in practical measurements due to a local violation of some assumptions, noise, outliers in the received signals, and many other reasons. Multiple estimates for each STARS-measured characteristic for each 78.6-s interval were considered as random samples of the characteristic which belong to the same statistical ensemble, and the scatter between the samples was interpreted as the sampling error. The mean $\bar{\psi}$ and the RMS $\mu_{\psi}$ values were estimated over 21 samples for all STARS-measured characteristics $\psi=\langle U\rangle,\langle V\rangle,\left\langle u^{2}\right\rangle$, etc. The means $\bar{\psi}$ are presented in all figures as the STARSmeasured values, and $\bar{\psi} \pm \mu_{\psi}$ are presented in the time series plots as the STARS-measured values with the measurement errors.

One can see a reasonable agreement in the mean winds measured by STARS and HAD, as well as measured by SA and DBS techniques. The HAD scatter is larger than that of STARS, although it is expected. As explained above, the STARS results for each 78.6-s interval are averages over 21 estimates for the interval. The current HAD analysis was carried out for only one triangle, the baselines $(1,2),(2,3)$, $(1,3)$, therefore, only three estimates for $\langle U\rangle$ and $\langle V\rangle$ were obtained with Eq. (21). Multiple estimates from actual and combined receiving antennas can also be used with the HAD technique for decreasing the scatter; e.g. Zhang et al. (2003).

One should note that the STARS measurement errors are the RMS rather than the peak values; the latter are about 3 times larger. Therefore, the existence of some of the HAD and DBS results outside the STARS error bars do not necessarily reflect significant disagreement with STARS. Furthermore, differences between individual estimates for $\langle U\rangle$ and $\langle V\rangle$ by different techniques of up to $10 \mathrm{~m} / \mathrm{s}$ seem quite natural and do not appear overly large for the relatively short averaging time in the present experiment.

One can also see that both the scatter in the HAD results, and the STARS measurement errors are much larger 

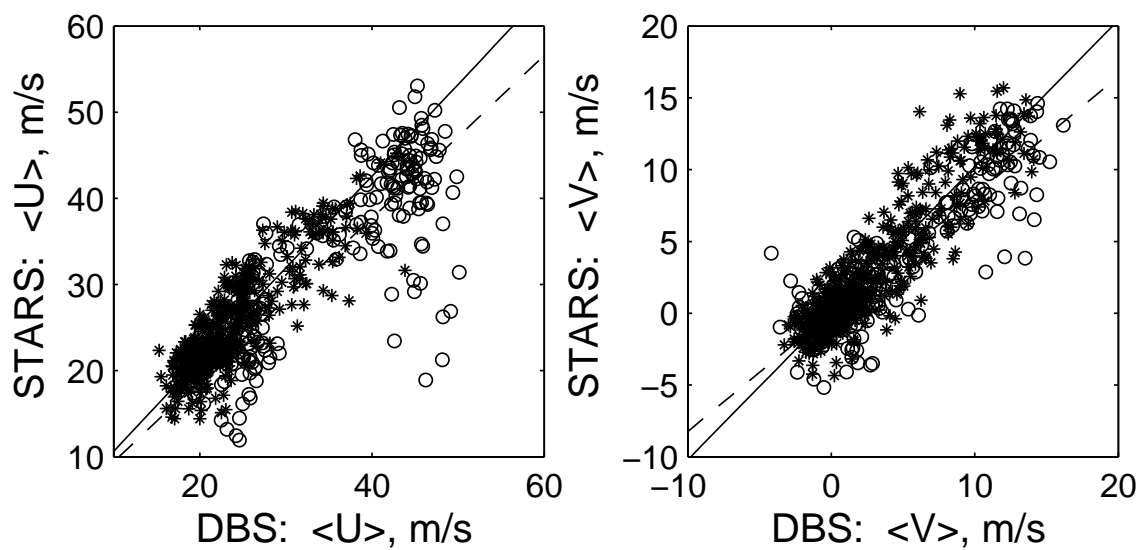

Fig. 5. Comparison of the mean horizontal wind speed components produced by STARS and DBS techniques at height from $5 \mathrm{~km}$ to $11 \mathrm{~km}$ above the MUR on 24 April 2002 at 19:30:00-22:00:00 LT. (1) SNR $\geq 3 \mathrm{~dB}$ : the results are shown by asterisks and solid lines. $\langle U\rangle: s=1.06$, $e=2.88 \mathrm{~m} / \mathrm{s}$. $\langle V\rangle: s=1.02, e=1.82 \mathrm{~m} / \mathrm{s}$. (2) $\mathrm{SNR}<3 \mathrm{~dB}$ : the results are shown by circles and dashed lines. $\langle U\rangle: s=0.94, e=6.00 \mathrm{~m} / \mathrm{s}$. $\langle V\rangle:$ $s=0.82, e=2.29 \mathrm{~m} / \mathrm{s}$.
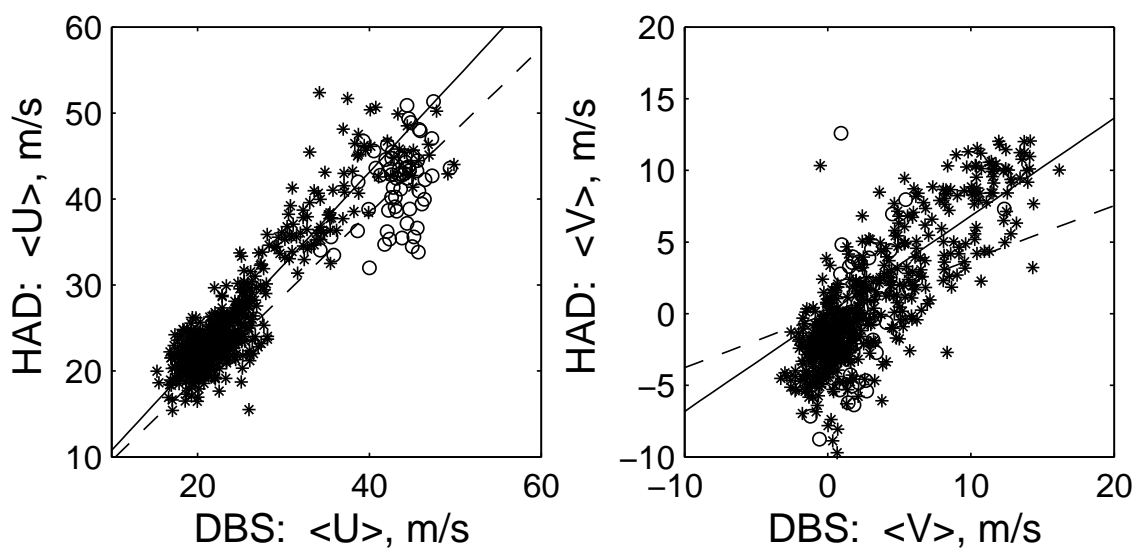

Fig. 6. The same as in Fig. 5 for HAD and DBS techniques. (1) $\mathrm{SNR} \geq 3 \mathrm{~dB}$ : $\langle U\rangle: s=1.08, e=2.96 \mathrm{~m} / \mathrm{s}$. $\langle V\rangle: s=0.68, e=2.66 \mathrm{~m} / \mathrm{s}$. (2) $\mathrm{SNR}$ $<3 \mathrm{~dB}:\langle U\rangle: s=0.96, e=4.24 \mathrm{~m} / \mathrm{s}$. $\langle V\rangle: s=0.38, e=3.76 \mathrm{~m} / \mathrm{s}$.

at $z_{o}=7.5 \mathrm{~km}$ than those at $5.1 \mathrm{~km}$, although the agreement of SA techniques with DBS still remains qualitatively reasonable. The degrading quality of the SA and DBS estimates with decreasing SNR is natural. One can further note that SA estimates are scattered around the DBS results, that is the former values may be either smaller or larger than the latter.

A point-to-point comparison of the mean wind speed components $\langle U\rangle$ and $\langle V\rangle$ produced by SA techniques with those produced by DBS is presented in Figs. 5 and 6; all results at $z_{o}=5-11 \mathrm{~km}$ are shown in these figures. The results are separated into two subsets: those for the DBS-estimated $\mathrm{SNR} \geq 3 \mathrm{~dB}$, and otherwise. The best linear fit (with no offset) was calculated separately for each subset and shown by solid and dashed lines, respectively. The fit is characterized by the slope $s$ and the RMS difference $e$ between the SA and DBS results; the values of $s$ and $e$ are presented in the figure captions. In calculating the best fit, the DBS and SA-measured winds were considered as independent and dependent variables, respectively. It is consistent with the initial choice of DBS technique as the "independent truth" for testing SA techniques.

One can see in Figs. 5 and 6 that the easterly mean winds $\langle U\rangle$ produced by the SA techniques are in fair agreement with those produced by the DBS technique. Moreover, the SA techniques agree fairly well between themselves: both the STARS and HAD slopes and the RMS errors for $\langle U\rangle$ at SNR $\geq 3 \mathrm{~dB}$ are very close to each other, that is $s=1.02$ and 1.08 , and $e=1.82 \mathrm{~m} / \mathrm{s}$ and $2.96 \mathrm{~m} / \mathrm{s}$ for STARS and HAD, respectively. One could note that the number of data points for HAD is smaller than that for STARS, which is due to the $\mathrm{HAD}$ data rejection. It is also seen in these figures that the agreement between SA and DBS techniques degrades dramatically when SNR decreases. 
Figure 5 shows that the STARS-produced northerly mean winds $\langle V\rangle$ are in fair agreement with those by the DBS technique, while agreement between HAD and DBS estimates of $\langle V\rangle$ in Fig. 6 is quite poor. The result is expected because typical values of $\langle V\rangle$ in the present experiment are very small. For small along-baseline winds, the peak of the cross CF is shifted considerably away from zero-lag, and thus the intercept of auto and cross CF, on which wind estimates depend, occurs at a low CF magnitude. The auto and cross CF widths are strongly reduced by turbulent fluctuations, further reducing the CF magnitude at the intercept lag. These factors lead to degrading accuracy of small along-baseline wind component estimates with HAD.

\subsection{Turbulence measurements}

Characteristics of turbulence were retrieved from the MUR signals in the SA mode with STARS and HAD techniques at the averaging time $T_{a v}=78.6 \mathrm{~s}$ while the spectrum width was estimated in the DBS mode at $T_{a v}=58.4 \mathrm{~s}$. The HAD estimates for $\left\langle w^{2}\right\rangle$ were obtained with Eqs. (15), (16), (19), and (20), and the STARS estimates for $\left\langle w^{2}\right\rangle,\left\langle u^{2}\right\rangle,\left\langle v^{2}\right\rangle$, and $\langle u v\rangle$ were obtained with Eqs. (4), (5), (12), and (13). Below $\sigma_{\phi}=\sqrt{\left\langle\phi^{2}\right\rangle}, \phi=u, v$, or $w$ denote the standard deviation of the turbulent velocity components.

The STARS-measured variances of turbulent velocity components were rejected for $\left\langle\phi^{2}\right\rangle \leq 0$, and the horizontal momentum flux was rejected for $|\langle u v\rangle|>\left(\sigma_{u} \sigma_{v}\right)$. Similarly, the negative values of $\left\langle w^{2}\right\rangle$ were rejected for the DBS and HAD techniques. It is well-known that the Doppler spectral width for the MUR is heavily affected by the beam broadening, such that at horizontal wind speeds of greater than approximately $40 \mathrm{~m} / \mathrm{s}$, it is often no longer possible to extract the spectral width due to turbulence (e.g. Hocking, 1987; Fukao et al., 1994; Furumoto and Tsuda, 2001). We tried to implement the beam-broadening corrections but it led to numerous negative values of $\left\langle w^{2}\right\rangle$. For this reason, the uncorrected spectral width is presented below.

Typical vertical profiles of turbulence characteristics are presented in Fig. 7. One can see that the STARS and HAD estimates for $\sigma_{w}$ are in a fair agreement at $z_{o} \leq 11 \mathrm{~km}$ while the agreement is poorer at low SNR. As expected, the DBSproduced values of $\sigma_{w}$ are much larger than those produced by SA techniques due to the beam broadening.

The beam broadening strongly affects the Doppler spectral width due to the underlying physical basis, the Doppler effect (e.g. Hocking, 1983a, 1987). The Doppler spectrum is a histogram of projections of the speed of individual scatterers on their lines of sight. For a broad transmitted beam, the lines of sight inside the beam are non-parallel to each other. The mean scatterers' motions, both along the beam centerline, and normal to the centerline increase the spectral width independent of turbulent motions. Therefore, the beam broadening affects the spectral width directly and heavily; it is an intrinsic feature of the Doppler effect.
This is not the case for SA techniques which have a different physical basis; see Sect. 4. Although the spectral width $\sigma_{t}$ could be related to the standard deviation of the vertical turbulent velocity $\sigma_{w}$ in Eq. (20), the relation is not unique and should not be interpreted as the equivalence between the two values. Indeed, CF of received signals (14) is the integral over the illuminated volume of the product of the spectral sampling function and the variance spectrum of the refractive index irregularities; e.g. DLH (pp. 163-164). The latter strongly depends on measurement conditions and is never known in practice. A formal relation between the spectral width and the width of CF can be obtained only by assuming a specific functional form and parameters of the spectral sampling function and the variance spectrum of the irregularities (e.g. DLH, Sects. 3, 4), and it could be considered only qualitatively rather than quantitatively.

To estimate the beam-broadening effect on the SAproduced values of $\left\langle w^{2}\right\rangle$, one can consider the coefficient $d_{\text {auto }}$ in Eq. (5). Following PPa, Eq. (12) for the coefficient contains only significant terms while a complete expression can be presented as follows:

$d_{\text {auto }}\left(\boldsymbol{x}_{a, k}\right)$
$=\frac{32 \pi^{2} \delta t^{2}}{\lambda^{2}}\left[\left\langle w^{2}\right\rangle+\frac{\theta^{2}}{\alpha^{2}}\left(\left\langle u^{2}\right\rangle+\left\langle v^{2}\right\rangle+\langle U\rangle^{2}+\langle V\rangle^{2}\right)\right]$,

where $\theta=\sigma / R=\gamma \lambda / D$ is the angular width of the transmitted beam. This equation was derived using only Assumptions $1 \mathrm{~S}-4 \mathrm{~S}$ and the first part of $5 \mathrm{~S}$ in Sect. 2.1; no assumptions about specific features of a scattering medium were applied. Equation (33) shows that the width of auto SF is affected by variances of the horizontal turbulent velocities with a relative weight of $\theta^{2} / \alpha^{2}$, with respect to that of the vertical velocity. For UHF and VHF SA radars, $\alpha^{2}$ varies typically from approximately 1.15 to 1.5 , and the beam width $\left(-3 \mathrm{~dB}\right.$ points) varies from approximately $3.5^{\circ}$ to $9^{\circ}$. Note that $\theta$ in Eq. (38), as well as $\sigma_{r}, \sigma$, and $\sigma_{a}$ in Sect. 2, are 2.36 times smaller than those estimated through $-3 \mathrm{~dB}$ points; see DLH (p. 160). For the MU radar, $\theta=0.0266$ radians, and $\alpha^{2}=1.28$ for the receiver configuration in Fig. 1, hence $\theta^{2} / \alpha^{2} \approx 0.00055$. Therefore, the beam-broadening effect on the STARS-produced variance $\left\langle w^{2}\right\rangle$ is negligibly small. As shown in Sect. 2.3, Eq. (12) for measuring $\left\langle w^{2}\right\rangle$ with STARS is similar to Eq. (20) for the HAD method, and a similarity between HAD and FCA is shown by Holloway et al. (1997a). Therefore, the above conclusion about the negligibly small effect of the beam broadening on the values of $\left\langle w^{2}\right\rangle$ produced by STARS with UHF and VHF SA radars is valid for the HAD and FCA techniques as well.

However, the above conclusion is rigorously valid only when assumptions $1 \mathrm{~S}-5 \mathrm{~S}$ for STARS and $1 \mathrm{H}-8 \mathrm{H}$ for HAD are satisfied. When the beam width increases, the validity of assumptions about statistical homogeneity becomes rather questionable, especially in the presence of intensive gravity waves. Therefore, the beam broadening could affect the SAmeasured values of $\left\langle w^{2}\right\rangle$ indirectly through violation of the underlying assumptions. The effect should not be noticable 

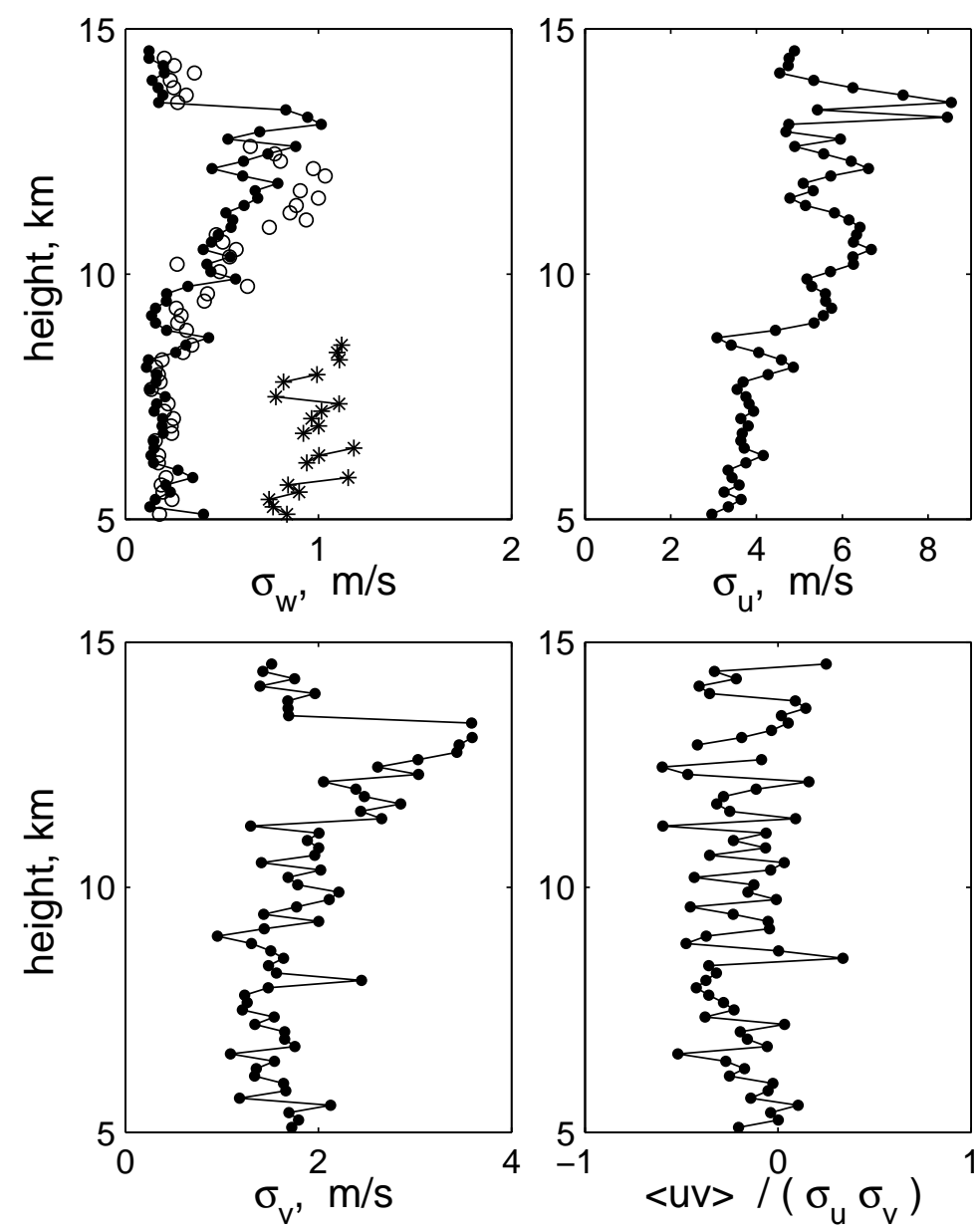

Fig. 7. The vertical profiles of turbulence characteristics above the MUR on 24 April 2002 at 21:51:26 LT. •, STARS; ॰, HAD; $*$, DBS.

for the MUR with a narrow transmitted beam (see Fig. 7 and Fig. 8 below) while it may be very significant for medium frequency radars with a very broad beam.

The STARS results show strong anisotropy of turbulence at all studied heights. A typical value of $\sigma_{w}$ is approximately $0.25 \mathrm{~m} / \mathrm{s}$ at $z_{o}<10 \mathrm{~km}$ while $\sigma_{u}$ and $\sigma_{v}$ are approximately $1.5 \mathrm{~m} / \mathrm{s}$ and $3.5 \mathrm{~m} / \mathrm{s}$, respectively. At $z_{o}=12 \mathrm{~km}$, the respective values are approximately $0.75 \mathrm{~m} / \mathrm{s}, 3 \mathrm{~m} / \mathrm{s}$, and $6 \mathrm{~m} / \mathrm{s}$. The non-dimensional momentum flux $\langle u v\rangle /\left(\sigma_{u} \sigma_{v}\right)$ reaches approximately -0.3 which is quite a large value.

Typical time series of turbulence characteristics at $z_{o} \leq 11 \mathrm{~km}$ are presented in Figs. 8 and 9. Again, the spectral width is much larger than the SA-produced $\sigma_{w}$; Fig. 8 . The agreement between STARS and HAD in measuring $\sigma_{w}$ is fair, and anisotropy of the STARS-measured standard deviations of turbulent velocities is very strong at all gates; Figs. 8 and 9. The measurement error in the STARS estimates for turbulence characteristics increases with height due to decreasing SNR.

A point-to-point comparison of the STARS and HADproduced standard deviation of the vertical turbulent velocity $\sigma_{w}$ at $z_{o}=5-11 \mathrm{~km}$ is presented in Fig. 10 . The results are separated into two subsets: those for the HAD-estimated
SNR $\geq 3 \mathrm{~dB}$, and otherwise. The best linear fit (with no offset) was calculated separately for each subset and shown by solid and dashed lines. The fit is characterized by the slope $s$ and the RMS difference $e$ between the STARS and HAD results; the values of $s$ and $e$ are presented in the figure caption. In calculating the best fit, the HAD and STARS-measured values of $\sigma_{w}$ were considered as independent and dependent variables, respectively. It is consistent with the initial choice of HAD as the traditional SA technique while STARS is a new technique whose potential is considered in the paper. One can see a fair agreement between the two SA techniques, although the STARS results for $\sigma_{w}$ are systematically smaller than the HAD results by approximately $20 \%$; the difference is discussed in Sect. 4.

\section{Discussion}

Using the MUR data collected in the SA and DBS modes, a reasonable agreement between the UCAR-STARS, HAD, and DBS-measured mean horizontal winds was found at sufficiently high SNR. Fair agreement between STARS and HAD in measuring the mean winds and variance of the 

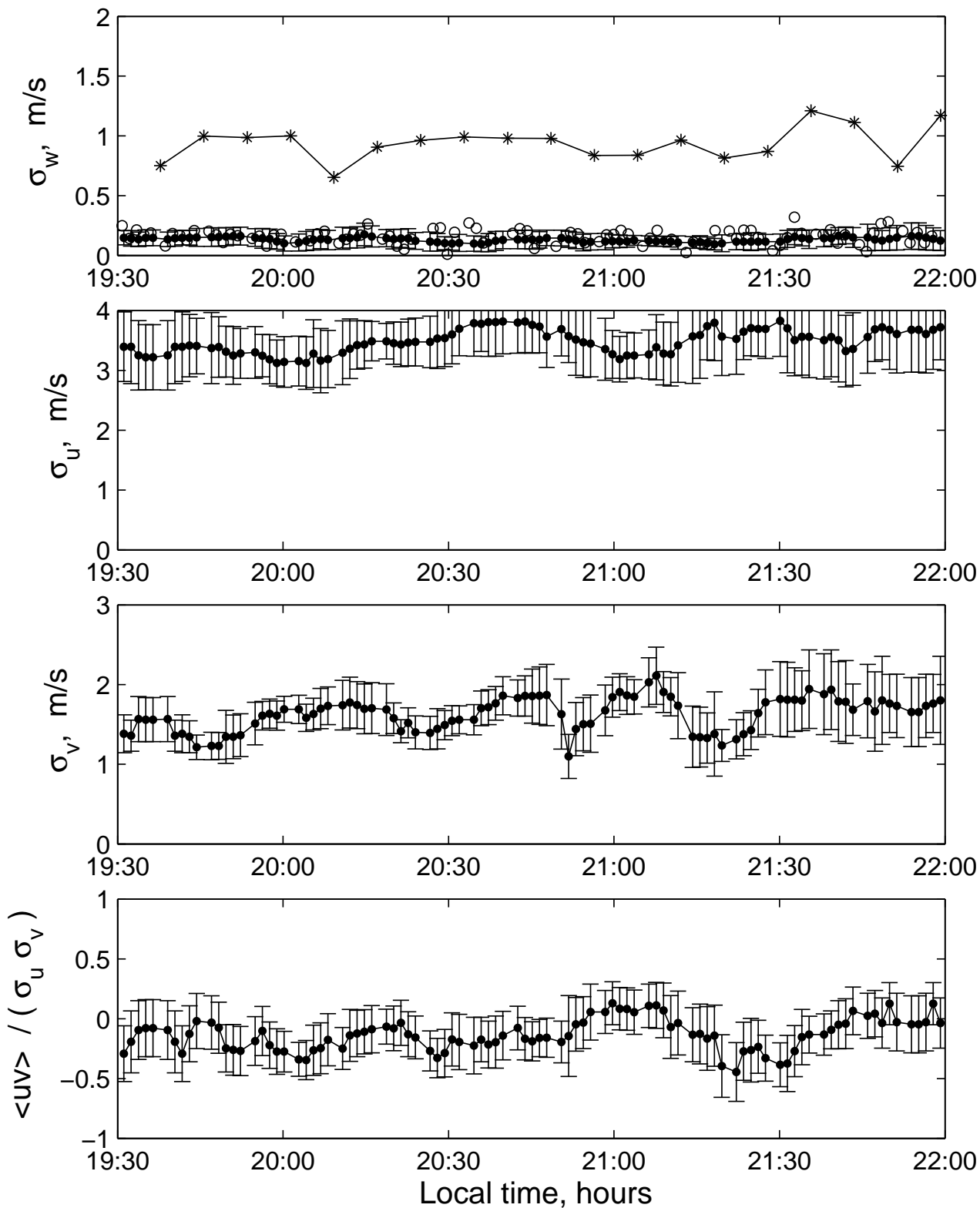

Fig. 8. Time series of turbulence characteristics on 24 April 2002 at $z_{o}=5.4 \mathrm{~km}$. $\bullet$ STARS; $\circ$, HAD; $*$, DBS.

vertical turbulent velocity seems to be expected because the considered SA techniques are related to each other; Sect. 2.3. However, the relations between the second order CF and SF do not indicate the equivalence between STARS and HAD (or any other) CF-based SA technique. Below we show that the CF and SF-based SA techniques are conceptually different, in spite of being formally related to each other for a particular case of the second-order functions.

CF can be applied only to the globally statistically stationary random processes. Real physical processes are almost never globally stationary while practically any process can be safely considered as being the locally statistically stationary (e.g. Tatarskii, 1971, chap. 1A; Monin and Yaglom, 1975, Sect. 13). Another term with the same meaning is a random process with statistically stationary increments. To quote
Tatarskii (1971, p. 16): "The structure function is a fundamental characteristic of a random process with stationary increments, and replaces the ideal of a correlation function." The word "ideal" refers to a globally stationary process that almost never exists in reality, especially in the atmosphere. The basic STARS Assumption 1S in Sect. 2.1 about a local stationarity is much less restrictive than the basic HAD Assumption $1 \mathrm{H}$ in Sect. 2.2 about a global stationarity.

Another remarkable theoretical feature of SF is the presence of a small parameter $\tau \rightarrow 0$. The small parameter always significantly simplifies a physical task by both leading to the asymptotically exact solutions, and requiring a smaller number of less restrictive assumptions (e.g. Migdal, 1977). Indeed, the STARS Assumptions $2 \mathrm{~S}$ and $3 \mathrm{~S}$ in Sect. 2.1 are the same as the HAD Assumptions $2 \mathrm{H}$ and $3 \mathrm{H}$ in Sect. 2.2. 

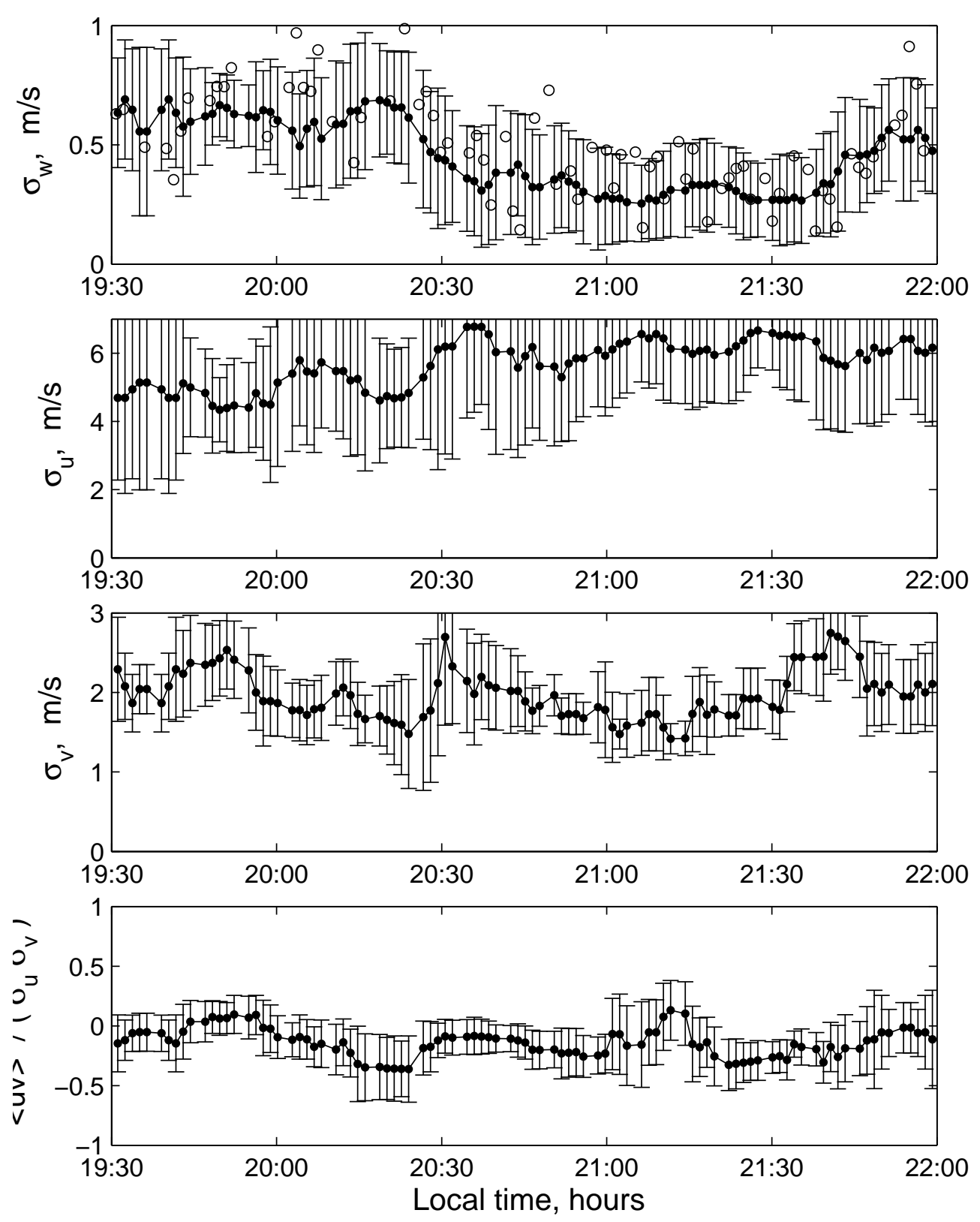

Fig. 9. The same as in Fig. 8 at $z_{o}=10.35 \mathrm{~km}$.

However, STARS requires only two additional Assumptions $4 \mathrm{~S}$ and $5 \mathrm{~S}$ for deriving operational equations for turbulence characteristics $\left\langle w^{2}\right\rangle,\left\langle u^{2}\right\rangle,\left\langle v^{2}\right\rangle$, and $\langle u v\rangle$. At the same time, HAD requires five much restrictive additional Assumptions $4 \mathrm{H}-8 \mathrm{H}$ for estimating the only turbulence characteristic $\left\langle w^{2}\right\rangle$.

$\mathrm{CF}$ characterizes fluctuations of a random process at all scales but mainly at the large ones of the order of the process's integral time scale $T_{\text {cor }}$. Fluctuations at large scales are heavily affected by external conditions, and the functional form of CF can never be universal (e.g. Townsend, 1956, Sects. 1.8, 1.9). In particular, the Gaussian functions (15) and (16) in the HAD technique are merely good approximations near the peak values of the auto and cross CF; the rigorous validity of Eqs. (15) and (16) over a wide range of $\tau$ would be more an exception than a rule in practical measurements.

SF characterizes fluctuations at small scales $\tau \ll T_{\text {cor }}$. The small-scale fluctuations of random processes are typically quite universal (e.g. Tatarskii, 1971, chap. 1A), and the decompositions (4) and (5) for cross and auto SF are valid almost always in practical measurements. On the other hand, $\mathrm{CF}$ is a more general characteristic of a random process than SF because it describes all scales. Indeed, Eqs. (29)-(32) express coefficients $d_{0}, d_{1}, d_{2}$, and $d_{\text {auto }}$ in Eqs. (4) and (5) for cross and auto SF using Eqs. (15)-(20) for cross and auto CF. The reverse operation, expressing coefficients $c_{0}, c_{1}, c_{2}$, and $c_{a u t o}$ in Eqs. (15) and (16) for CF using equations for SF is 


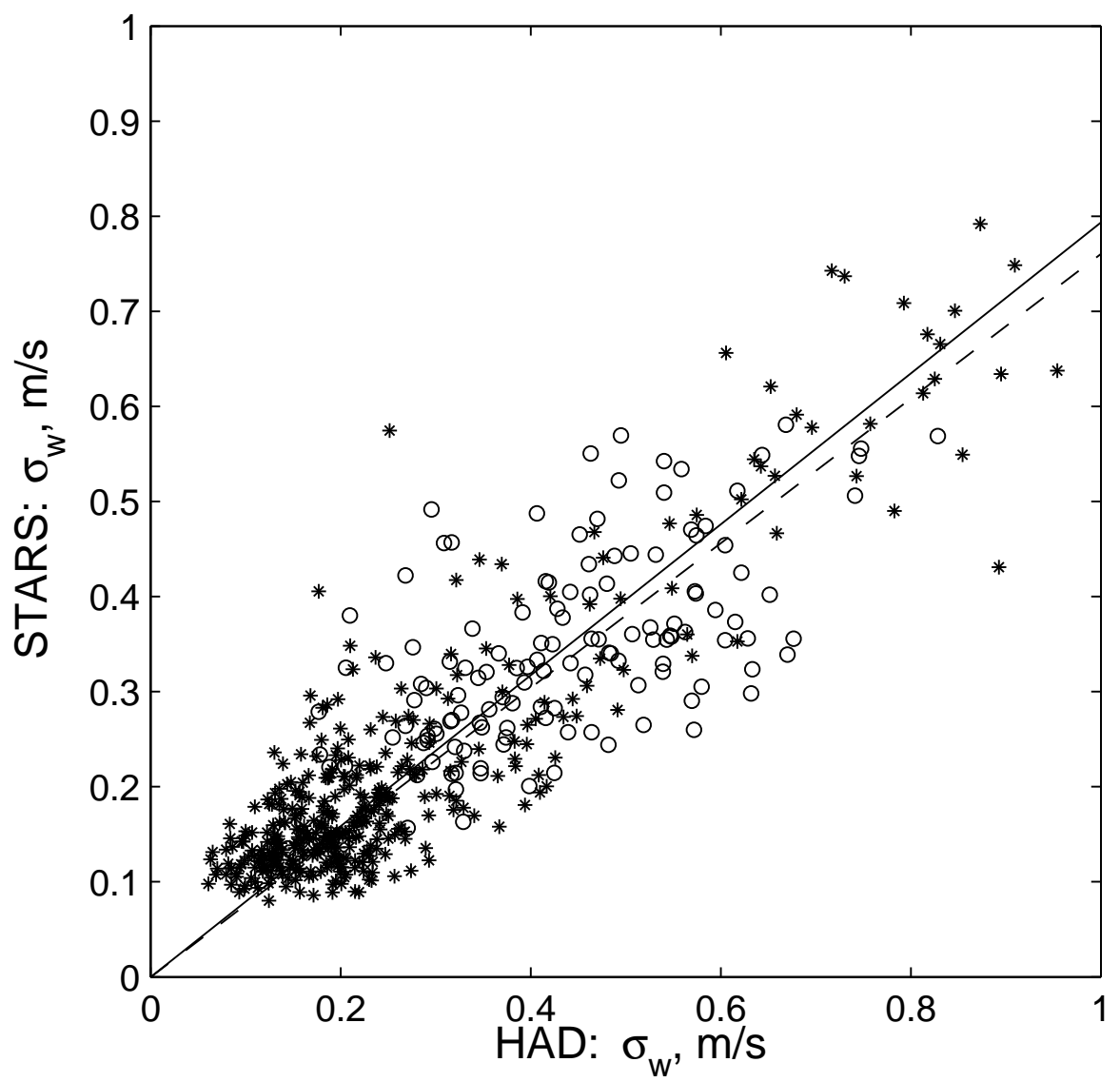

Fig. 10. Comparison of the STARS and HAD-produced standard deviation of the vertical turbulent velocity at height from $5 \mathrm{~km}$ to $11 \mathrm{~km}$ above the MUR on 24 April 2002 at 19:30:00-22:00:00 LT. (1) SNR $\geq 3 \mathrm{~dB}$ : the results are shown by asterisks and solid lines. $s=0.79$, $e=0.06 \mathrm{~m} / \mathrm{s}$. (2) $\mathrm{SNR}<3 \mathrm{~dB}$ : the results are shown by circles and dashed lines. $s=0.76, e=0.09 \mathrm{~m} / \mathrm{s}$.

impossible because the latter are valid only at $\tau \rightarrow 0$.

Only the second order SF are considered in this paper. As shown in PPa, SF of any order $p \geq 2$ can be derived for a pair of received signals and applied to practical measurements while CF at only $p=2$ are used in SA techniques. It is important that equations for $\mathrm{SF}$ at $p>2$, for example, those for estimating the higher-order turbulence characteristics $\left\langle w^{3}\right\rangle$, $\left\langle u^{3}\right\rangle,\left\langle v^{3}\right\rangle,\left\langle w^{4}\right\rangle,\left\langle u^{4}\right\rangle,\left\langle v^{4}\right\rangle$, and so on, can be derived using the same Assumptions $1 \mathrm{~S}-5 \mathrm{~S}$ as at $p=2$; see $\mathrm{PPa}$ and $\mathrm{PPb}$.

Therefore, SF is a more powerful theoretical tool than CF. Again, quoting Tatarskii (1971, p. 17): "For stationary random processes the structure function $D_{f}(\tau)$ can be used on an equal footing with the correlation function; in some respects it is even more expedient. Indeed, when we approach a random process whose stationarity is not evident beforehand, a better policy is to construct its structure function, and not the correlation function. In practice, the construction of structure function is always more reliable, since $D_{f}(\tau)$ is not affected by errors in the mean $\langle f(t)\rangle$." Here $f(t)$ is a random process of an arbitrary physical nature, and $D_{f}(\tau)=\left\langle[f(t)-f(t+\tau)]^{2}\right\rangle$.

However, the major difference between CF and SF-based SA techniques is not in the above theoretical details but rather in the physical concept beneath the techniques. The cross CF (14) describes the similarity between signals from two receivers $E\left(\boldsymbol{x}_{a, k}, t\right)$ and $E\left(\boldsymbol{x}_{a, k}+\Delta \boldsymbol{x}_{m k}, t+\tau\right)$ at all temporal separations $-\infty<\tau<\infty$. For example, the maximum value $\left|C\left(\Delta \boldsymbol{x}_{m k}, \tau_{p}\right)\right|_{\text {max }}$ defines the lag $\tau_{p}$ for which the signal $E\left(\boldsymbol{x}_{a, k}+\Delta \boldsymbol{x}_{m k}, t+\tau_{p}\right)$ is the mostly similar to the signal $E\left(\boldsymbol{x}_{a, k}, t\right)$. A specific technique for relating CF for received signals (that is, $\mathrm{CF}$ of the diffraction pattern on the ground) to characteristics of a scattering medium could be very simple as in the plane drift method for the "frozen" field of scatterers (e.g. Briggs et al., 1950, Hocking, 1983b, Briggs, 1984), or rather sophisticated, as in the FCA and HAD techniques. The latter could take into account spatial/temporal decorrelation due to turbulence, anisotropy of scatterers, and many other important aspects of a scattering medium, as well as specific radar parameters (e.g. Briggs, 1984; Hocking, 1989; Hocking et al., 1989; Briggs and Vincent, 1992; DLH; Holloway et al., 1997a). However, the physical concept underneath all CF-based techniques is uniquely defined by the CF as a mathematical tool: revealing the similarity between signals at all scales. The concept is the following: tracking the diffraction pattern and its changes, and, therefore, tracking a scattering medium and its changes in the illuminated volume. 
The cross SF (2) describes the difference between the signals $S\left(\boldsymbol{x}_{a, k}, t\right)$ and $S\left(\boldsymbol{x}_{a, k}+\Delta \boldsymbol{x}_{m k}, t+\tau\right)$. The increment $S\left(\boldsymbol{x}_{a, k}, t\right)-S\left(\boldsymbol{x}_{a, k}+\Delta \boldsymbol{x}_{m k}, t+\tau\right)$ is a filter that extracts fluctuations with spatial and temporal scales $\left|\Delta \boldsymbol{x}_{m k}\right|$ and $\tau$, respectively. At $\tau \rightarrow 0$, the increment $\left[S\left(\boldsymbol{x}_{a, k}, t\right)-S\left(\boldsymbol{x}_{a, k}, t+\tau\right)\right] / \tau$ tends to the temporal derivative $\partial S\left(\boldsymbol{x}_{a, k}, t\right) / \partial t$. Similarly, the increment $\left[S\left(\boldsymbol{x}_{a, k}, t\right)-S\left(\boldsymbol{x}_{a, k}+\Delta \boldsymbol{x}_{m k}, t\right)\right] /\left|\Delta \boldsymbol{x}_{m k}\right|$ at $\left|\Delta \boldsymbol{x}_{m k}\right| \rightarrow 0$ tends to the spatial derivative $\partial S\left(\boldsymbol{x}_{a, k}, t\right) / \partial\left|\boldsymbol{x}_{a, k}\right|$ of the signal $S\left(\boldsymbol{x}_{a, k}, t\right)$, with the receiver center $\boldsymbol{x}_{a, k}$ being moved in the direction $\Delta \boldsymbol{x}_{m k}$; see PPa for details. Therefore, a SF-based technique is intrinsically differential while a derivative characterizes the rate of change in the function. Independent of specific technique for relating SF for received signals to characteristics of a scattering medium, the physical concept underneath SF-based techniques is uniquely defined by the SF as a mathematical tool: revealing the difference between signals at small scales $\left|\Delta \boldsymbol{x}_{m k}\right|$ and $\tau \rightarrow 0$. The concept is the following: evaluating the rates of spatial and temporal changes in the diffraction pattern, and, therefore, evaluating the rates of spatial and temporal changes in a scattering medium in the illuminated volume.

The above considerations show that CF and SF-based techniques utilize conceptually different physical features of the diffraction pattern and a scattering medium. Some practical consequences from the conceptual difference between the techniques are discussed below.

To increase the sensitivity of the measurements with $\mathrm{CF}$, one should apply a CF-based technique at the range of separations $\left|\Delta \boldsymbol{x}_{m k}\right|$ and $\tau$ close to the maximum gradient of the functions. For the Gaussian CF (15) and (16), the maximum gradient is at such spatial and temporal separations where cross and auto $\mathrm{CF}$ are around 0.5. To ensure high measurement accuracy, "it is undesirable to work with very high values of correlation" (Briggs, 1984, p. 174), therefore, the receiver separation $\left|\Delta \boldsymbol{x}_{m k}\right|$ should not be too small. Another reason for applying sufficiently large $\left|\Delta \boldsymbol{x}_{m k}\right|$ with a CF-based SA technique is referred to as the triangle effect (e.g. Meek, 1980b; Holdsworth and Reid, 1997). Typical separation between the antenna centers for applying CFbased SA techniques is usually chosen in such a way as to ensure $\left|C\left(\Delta \boldsymbol{x}_{m k}, 0\right)\right| \approx 0.2-0.7$ (e.g. Awe, 1964; Vincent, 1984; Hocking et al., 1989; Meek and Manson, 2001). The range of temporal separations for fitting Eqs. (15) and (16) to experimental data is typically chosen in such a way as to cover CF from approximately 0.05 , and higher. Tens, or even hundreds of data points including rather large values of $\tau$, are employed into the fitting.

There are two CF-based techniques which apply only cross $\mathrm{CF}$ at $\tau \rightarrow 0$ for measuring the mean horizontal winds (but no turbulence): a slope at zero lag by Lataitis et al. (1995), and the cross-correlation ratio by Zhang et al. (2003). The latter is a straightforward modification of the HAD technique for decreasing the measurement error by excluding auto $\mathrm{CF}$ from the wind measurements. Although the techniques could decrease the measurement uncertainties at some conditions, they are still identical conceptually to other CF-techniques in that they track a motion of the diffraction pattern as a whole.

On the contrary, the SF requires as small separations as possible to approximate the derivatives. Indeed, the smaller $|\tau|$ and $\left|\Delta \boldsymbol{x}_{m k}\right|$ are, the better one can estimate the derivatives $\partial S\left(\boldsymbol{x}_{a, k}, t\right) / \partial t$ and $\partial S\left(\boldsymbol{x}_{a, k}, t\right) / \partial\left|\boldsymbol{x}_{a, k}\right|$, and the more accurate are the SF-based measurements; see PPa for details. For this reason, only a few separations, typically not more than $\tau= \pm \delta t, \pm 2 \delta t$, and $\pm 3 \delta t$, are used in STARS. The combined signals are employed in STARS not only for obtaining more estimates for each measured characteristic but mainly for decreasing a spatial separation between the receiver centers. Therefore, CF and SF-based techniques use the different parts of the functions in practical measurements: those at large and small separations, respectively.

Utilizing different physical features of the diffraction pattern, and using different ranges of temporal separations, CF and SF-based techniques are differently affected by noise. Theoretically, CF are unaffected by white noise (except for the auto CF at zero lag) while they are strongly affected by any noise with a finite temporal scale, especially with a large one, such as ground clutter. As any differential value, both auto and cross SF are strongly affected by any noise with a small temporal scale, at all lags, in particular by a white noise. On the other hand, SF is not sensitive to noise with a large temporal scale such as ground clutter, or hard targets (PPa, Sect. 4; Praskovskaya et al., 2003). This feature can be easily understood from the definition of SF, Eq. (2). A statistical difference between two signals at separation $\tau$ cannot "sense" a process with a temporal scale $T_{\text {cor }} \gg|\tau|$ because such a process is merely filtered by the increment. In practice, noise with a small temporal scale affects SFbased techniques much stronger than the CF-based ones. Indeed, fitting CF to Eqs. (15) and (16) over many separations $\tau_{\min } \leq \tau \leq \tau_{\max }$, one merely "smoothes" CF with a temporal scale $T_{s}=\tau_{\max }-\tau_{\min }$, and filters the processes with smaller than $T_{s}$ scales. On the contrary, SF is fitted only at $|\tau| / \delta t \leq 3$, and all processes with the scales below at least $10 \delta t$ strongly affect the results.

As noted in Sect. 2.1, the coefficients $d_{0}\left(\Delta \boldsymbol{x}_{m k}\right)$, $d_{1}\left(\Delta \boldsymbol{x}_{m k}\right), d_{2}\left(\Delta \boldsymbol{x}_{m k}\right)$, and $d_{\text {auto }}\left(\boldsymbol{x}_{a, k}\right)$ in the cross and auto SF (4) and (5) can be obtained from Eqs. (14)-(20) for the cross and auto $\mathrm{CF}$ at $\tau \rightarrow 0$. However, Eqs. (11) and (31) for the coefficient $d_{2}\left(\Delta \boldsymbol{x}_{m k}\right)$ and its CF-based estimate $\tilde{d}_{2}\left(\Delta \boldsymbol{x}_{m k}\right)$ differ by the term with $\left\langle u_{m k}^{2}\right\rangle$. This term describes the variation of the turbulent velocity along the baseline $\Delta \boldsymbol{x}_{m k}$ and plays a very important role in the STARS method. It leads to the operational Eq. (13) for estimating the variation of the horizontal turbulent velocities and the flux $\langle u v\rangle$. Similar terms at $p>2$ would lead to equations for estimating $\left\langle u^{4}\right\rangle$, $\left\langle v^{4}\right\rangle,\left\langle u^{2} v^{2}\right\rangle$, and so on. One can see from Eqs. (11)-(13) that measuring characteristics of the horizontal turbulent velocities with STARS is possible because $d_{2}\left(\Delta \boldsymbol{x}_{m k}\right) \neq d_{a u t o}\left(\boldsymbol{x}_{a, k}\right)$. The term $\left\langle u_{m k}^{2}\right\rangle$ appears in the derivation of Eq. (11) from Eq. (7) as $\left\langle U_{i, m k}^{2}(t)\right\rangle=\left\langle U_{i, m k}\right\rangle^{2}+\left\langle u_{i, m k}^{2}\right\rangle$. From a physical point of view, the term $\left\langle U_{i, m k}^{2}(t)\right\rangle$ reflects a rather obvious fact: the rates of spatial and temporal changes in the 
diffraction pattern along $\Delta \boldsymbol{x}_{m k}$ are proportional to the square of the instantaneous velocity component along the baseline. This can be easily obtained from simple dimensional considerations, and it is proven rigorously in PPa for any scalar random field.

At the same time, the HAD coefficients $c_{2}\left(\Delta \boldsymbol{x}_{m k}\right)$ and $c_{\text {auto }}\left(\boldsymbol{x}_{a, k}\right)$ in the cross and auto CF are identical; Eqs. (19) and (20). It is noteworthy that Eq. (19) is the major operational equation in the FCA technique (Briggs, 1984, p. 176). One can see that $c_{2}\left(\Delta \boldsymbol{x}_{m k}\right)$ does not contain $\left\langle u_{m k}^{2}\right\rangle$ which is quite natural from a physical point of view. Indeed, cross $\mathrm{CF}\left|C\left(\Delta \boldsymbol{x}_{m k}, \tau\right)\right|$ reveals a statistical similarity between the signals $E\left(\boldsymbol{x}_{a, k}, t\right)$ and $E\left(\boldsymbol{x}_{a, k}+\Delta \boldsymbol{x}_{m k}, t+\tau\right)$; it tracks the diffraction pattern in its motion from $\boldsymbol{x}_{a, k}$ to $\boldsymbol{x}_{a, k}+\Delta \boldsymbol{x}_{m k}$. It is quite obvious that fluctuations $u_{m k}(t)$ along the baseline $\Delta \boldsymbol{x}_{m k}$ cannot affect the statistical similarity between the signals but only make $E\left(\boldsymbol{x}_{a, k}+\Delta \boldsymbol{x}_{m k}, t+\tau\right)$ more "blurring", that is only the mean speed $\left\langle U_{m k}\right\rangle$ can be detected while tracking motion of the pattern as a whole. For a formal explanation of the above statement, one should note that the term with $\left\langle U_{m k}\right\rangle^{2}+\left\langle u_{m k}^{2}\right\rangle$ in Eq. (11) for $d_{2}\left(\Delta \boldsymbol{x}_{m k}\right)$ is simulated by the term $c_{1}^{2}\left(\Delta \boldsymbol{x}_{m k}\right)$ in Eq. (31). The latter contains only $\left\langle U_{m k}\right\rangle$, Eq. (18). One can easily obtain from simple dimensional considerations that $c_{1}\left(\Delta \boldsymbol{x}_{m k}\right) \propto\left\langle U_{m k}\right\rangle+\left\langle u_{m k}\right\rangle$, although the last term is zero by definition. This is the place where the horizontal turbulent velocity formally disappears from the coefficient $c_{2}\left(\Delta \boldsymbol{x}_{m k}\right)$ in Eq. (15) for cross CF.

Therefore, the conceptual difference between CF and SF-based approaches to analyzing received signals for SA radars, namely tracking the diffraction pattern and evaluating the rates of changes in the pattern, respectively, leads to significant practical differences. One can potentially estimate the different order moments of all turbulent velocity components separately with the SF-based SA techniques while only $\left\langle w^{2}\right\rangle$ can be estimated with the CF-based techniques. Other characteristics, such as the turbulent kinetic energy, eddy dissipation rate, and so on, can be estimated with CF-based techniques only by assuming the isotropy, the dynamic equilibrium, or a specific functional form of the turbulence spectrum, and/or with other restrictive assumptions (e.g. Briggs, 1980; Hocking, 1983a, 1989; Hocking et al., 1989; DLH).

Let us consider the results for turbulence variations from the SF and CF methods in light of the above discussion. One could notice in Figs. 7-9 rather large values of $\left\langle u^{2}\right\rangle$ and $\left\langle v^{2}\right\rangle$ while the flow above the MUR at $z_{o}=5-11 \mathrm{~km}$ is practically laminar in most cases (e.g. Luce et al., 1999, 2000; Tsuda et al., 1986, 1997). Following PPa and PPb, the instantaneous velocities of each scatterer are described in this paper by Eq. (6) as the classic Reynolds decomposition into the mean and turbulent components. Decomposition (6) interprets any fluctuations with respect to the mean as being turbulent independent of their real physical nature. The same decomposition is used in the CF-based techniques (e.g. Briggs, 1980; DLH). Equation (6) is physically adequate in a rather turbulent atmospheric boundary layer. A reasonable agreement of the STARS-measured variances $\left\langle w^{2}\right\rangle,\left\langle u^{2}\right\rangle,\left\langle v^{2}\right\rangle$, and flux $\langle u v\rangle$ with those measured by the co-located sonic anemometer is reported in $\mathrm{PPb}$. However, Eq. (6) seems to be inadequate for describing a laminar flow with intensive gravity waves above the MUR. The waves in the horizontal direction with a period of approximately $1-1.5 \mathrm{~h}$ are clearly seen in all presented time series; Figs. 3, 4, 8, and 9. The wave propagation in the vertical direction with a scale of approximately $2 \mathrm{~km}$ can be observed in the vertical profiles in Figs. 2 and 7, although not so clear. One could speculate that the STARS-produced large values for the horizontal turbulent velocities $\left\langle u^{2}\right\rangle$ and $\left\langle v^{2}\right\rangle$ in Figs. 7-9 are caused by the wave motion rather than small-scale turbulence. The same could be the case for the HAD and STARS-produced variation of the vertical turbulent velocity $\left\langle w^{2}\right\rangle$. This speculation is consistent with systematically smaller values of the STARS-produced $\sigma_{w}$ than those produced by HAD; Fig. 10. Indeed, the waves have a large scale; only a small part of the wave-induced fluctuations affects the rate of changes in the diffraction pattern and is registered by $\mathrm{SF}$.

The CF registers all scales in the illuminated volume, that is the larger part of the wave-induced fluctuations, and the CF-produced $\sigma_{w}$ is larger than that produced by SF. Measurements of turbulence at high altitudes can become more adequate and physically meaningful if one replaces Eq. (6) by more explicit decomposition of the instantaneous velocity into the mean background velocity, the gravity wave perturbation velocity, and the turbulent velocity (e.g. Holdsworth and Reid, 1995, p. 1266).

It is not, however, clear whether one could be able to resolve unambiguously characteristics of the wave-induced and small-scale turbulent fluctuations with $\mathrm{CF}$, and/or SFbased approaches. Therefore, turbulence measurements at high altitudes should be taken with great caution, and their interpretation should incorporate as much knowledge about a specific scattering medium as possible. The STARSproduced variations $\left\langle u^{2}\right\rangle,\left\langle v^{2}\right\rangle$, and the flux $\langle u v\rangle$ are presented only for illustrating the UCAR-STARS potential towards comprehensive turbulence measurements with SA profiling radars.

\section{Summary}

SA methods for analyzing complex time series of signals from multiple receivers have become commonly used techniques to measure parameters of the atmosphere from the lower troposphere up into the ionosphere. Using either a heuristic approach in the time or frequency domain, or alternatively, a more rigorous approach assuming a model of the atmospheric scatterer medium, algorithms making use of correlation functions (FCA) or spectra (FSA) have been developed to estimate mean horizontal winds and vertical turbulent velocity, as well as, for the latter approach (HAD method), spatial scales of refractive index irregularities. A further signal analysis approach (UCAR-STARS), using structure functions as a basis from which to derive 
parameters of winds and turbulence, has recently been developed. This method need assume only local stationarity, whereas methods using $\mathrm{CF}$ or spectra require an assumption of global stationarity. This feature makes the application of the SF method more amenable to physical problems in the atmosphere. In this paper the SF-based method of data analysis is compared to the HAD method which is deemed the most rigorous CF-based analysis technique. Comparison of parameter estimates by the two methods is shown for data obtained from the MU radar.

Previous authors have derived the relationship between radar received complex signals at three or more antennas and atmospheric scatter using a volume scattering model, from which horizontal winds and characteristics of turbulence are obtained for the HAD and the STARS methods. In our notation, a scatterer was defined as a property of the atmosphere to which the radar is sensitive, namely refractive index fluctuations characterized by instantaneous locations, velocities and reflectivities. For the STARS method using only secondorder structure functions, which correspond to the secondorder correlation functions of the HAD method, a relationship between operational equations for the two methods is shown, based on standard assumptions about received signals. Furthermore, the necessary assumptions for the two methods were reproduced and compared qualitatively.

We presented the first experimental test of the UCARSTARS method with the MST profiling radar in the SA mode. The STARS-measured mean horizontal wind speed components were compared with those produced by the DBS method and the CF-based HAD SA technique. A reasonable agreement of the STARS results with those by DBS and HAD was found at high SNR. The STARS and HAD-produced variances of vertical turbulent velocity were in fair agreement, as well as affected by beam broadening in a different way than the DBS-produced spectral width, and to a much lesser degree. The STARS-measured horizontal turbulence characteristics $\left\langle u^{2}\right\rangle,\left\langle v^{2}\right\rangle$, and $\langle u v\rangle$ are also presented as an illustration of the method's potential, although we called for a caution in the interpretation of the results.

Turbulence results from the HAD method were systematically larger by about $20 \%$ compared to the STARS method estimates. We argued when using Reynolds decomposition to obtain turbulence estimates, that gravity waves occurring at altitude and visible in the data could affect the respective results from the two methods by contributing, by virtue of their large scales, more to the CF method estimates of the variance of vertical turbulent velocity than to the SF method estimates. A more exact knowledge of the specific scattering medium, combined with more explicit decomposition of the instantaneous velocity is required to improve turbulence estimates.

It was shown that $\mathrm{CF}$ and SF-based SA techniques are related to one other while they are conceptually different. The underlying physical concepts are tracking the diffraction pattern and its changes, and evaluating the rates of spatial and temporal changes in the pattern, respectively. Ideal spatial and time increments for CF methods, therefore, are much larger than for SF methods, which might lead to the preferential use of one or the other method for a given observed diffraction pattern. Due to this difference, the SF-based methods are much more strongly affected by noise with small temporal scale than the CF-based techniques, while noise with a large scale, such as ground clutter, affects CF much more strongly than SF.

The use of diffraction pattern similarity in CF methods compared to that of the rate of temporal and spatial change by SF methods means that only variance of vertical turbulent velocity $\left\langle w^{2}\right\rangle$ can be measured with CF-based techniques, unless rather restrictive additional assumptions are employed. SF-based techniques potentially allow for measuring moments of the order of $p \geq 2$ of all turbulent velocity components separately, such as $\left\langle w^{3}\right\rangle,\left\langle u^{3}\right\rangle,\left\langle v^{3}\right\rangle,\left\langle w^{4}\right\rangle,\left\langle u^{4}\right\rangle,\left\langle v^{4}\right\rangle$, $\left\langle u^{2} v^{2}\right\rangle$, and others.

Therefore, CF and SF-based SA techniques do not compete but rather complement each other. The UCAR-STARS method could become a useful alternative to the traditional CF and spectra-based data analysis techniques for SA radars, although extensive study of the technique is still needed.

Acknowledgements. The authors are deeply grateful to the reviewers for their valuable and constructive comments. Comments on the draft by C. Holloway are highly appreciated. NCAR is sponsored by the National Science Foundation (NSF). The first author (AP) was sponsored by the NCAR/RAP Director's fund, and the second author (EP) was sponsored by the NSF Grant ATM-0122877. The MU radar belongs to and is operated by the Radio Science Center for Space and Atmosphere (RASC) of Kyoto University. AP and EP were supported by RASC as a visiting professor and a visiting scientist, respectively, during the major part of this research. AP was supported by the International Centre for Theoretical Physics (Trieste, Italy) as a visiting professor during part of his work on this paper.

Topical Editor U.-P. Hoppe thanks A. Manson and another referee for their help in evaluating this paper.

\section{References}

Awe, O.: Effects of errors in correlation on the analysis of the fading of radio wave, J. Atmos. and Terr. Phys., 26, 1257-1271, 1964.

Briggs, B. H.: Radar observations of atmospheric winds and turbulence: a comparison of techniques, J. Atmos. and Terr. Phys., 42, 823-833, 1980.

Briggs, B. H.: The analysis of spaced sensor records by correlation techniques, MAP Handbook, 13, 166-186, 1984.

Briggs, B. H., Phillips, G. J., and Shinn, D. H.: The analysis of observations on spaced receivers of the fading radio signals, Proc. Phys. Soc. London, 63, 106-121, 1950.

Briggs, B. H. and Vincent, R. A.: Spaced-antenna analysis in the frequency domain, Radio Sci., 27, 117-129, 1992.

Brown, W. O. J., Fraser, G. J., Fukao, S., and Yamamoto, M.: Spaced antenna and interferometric velocity measurements with MF and VHF radars, Radio Sci., 30, 1281-1292, 1995a.

Brown, W. O. J., Fukao, S., Yamamoto, M., and Fraser, G. J.: Spaced antenna scattering scale and orientation measurements of the upper troposphere on the MU radar, J. Geomag. Geoelectr., 47, 1231-135, $1995 \mathrm{~b}$. 
Chau, J. L. and Balsley, B. B.: A statistical comparison of VHF techniques to study clear-air vertical velocities in the lower atmosphere using the Jicamarca radar, Radio Sci., 33, 1565-1583, 1998.

Chilson, P. B., Palmer, R. D., Larsen, M. F., and Ulbrich, C. W.: First observations of precipitation with a spatial interferometer, GRL, 12, 2409-2412, 1992.

Cohn, S. A, Brown, W. O. J., Martin, C. L., Susedik, M. S., Maclean, G., and Parsons, D. B.: Clear air boundary layer spaced antenna wind measurement with the multiple antenna profiler (MAPR), Ann. Geophys., 19, 845-854, 2001.

Cohn, S. A, Holloway, C. L., Oncley, S. P., Doviak, R. J., and Lataitis, R. J.: Validation of a VHF spaced antenna wind profiler for high-resolution boundary layer observations, Radio Sci., 32, 1279-1296, 1997.

Doviak, R. J., Lataitis, R. J., and Holloway, C. L. (DLH): Cross correlations and cross spectra for spaced antenna wind profilers, 1 , Theoretical analysis, Radio Sci., 31, 157-180, 1996.

Doviak, R. J. and Zrnić, D. S.: Doppler Radar and Weather Observation, Academic Press, 1993.

Fedor, L. S.: A statistical approach to the determination of threedimensional atmospheric drifts, J. Geophys. Res., 72, 5401$5415,1967$.

Fooks, G. F.: Ionospheric drift measurements using correlation analysis, Methods for computation and interpretation of results, J. Atmos. Terr. Phys., 27, 979-989, 1965.

Fukao, S. and Palmer, R. D.: Spatial and frequency domain interferometry using the MU radar: A tutorial and recent developments, J. Geomag. Geoelectr., 43, 645-666, 1991.

Fukao, S., Sato, T., Tsuda, T., Kato, S., Wakasugi, K., and Makihira, T.: The MU radar with an active phased array, 1, Antenna and power amplifiers, Radio Sci., 20, 1155-1168, 1985a.

Fukao, S., Sato, T., Tsuda, T., Kato, S., Wakasugi, K., and Makihira, T.: The MU radar with an active phased array, 2, In-house equipment, Radio Sci., 20, 1169-1176, 1985b.

Fukao, S., Yamanaka, M. D., Ao, N., Hocking, W. K., Sato, T., Yamamoto, M., Nakamura, T., Tsuda, T., and Kato, S.: Seasonal variability of vertical eddy diffusivity in the middle atmosphere, 1. 3-year observations by the Middle and Upper-atmosphere radar, J. Geophys. Res., 99 (D9), 18 973-18 987, 1994.

Furumoto, J. and Tsuda, T.: Characteristics of energy dissipation rate and effect of humidity on turbulence echo power revealed by MU radar-RASS measurements, J. Atmos. Terr. Phys., 63, 285294, 2001.

Hall, C. M., Manson, A. H., and Meek, C. E.: Seasonal variation of the turbopause: One year of turbulence investigation at $69^{\circ} \mathrm{N}$ by the Joint University of Troms $\varnothing /$ University of Saskatshewan MF radar. J. Geophys. Res., 103, 28 769-28 773, 1998.

Hassenpflug, G., Rao, P. B., Yamamoto, M., and Fukao, S.: MU radar spaced antenna observations with varying apertures: Scatterer and antenna contributions to the ground diffraction pattern, Radio Sci., 38, 1043-1053, 2003.

Hocking, W. K.: On the extraction of atmospheric turbulence parameters from radar backscatter Doppler spectra, I. Theory, J. Atmos. Terr. Phys., 45, 89-102, 1983a.

Hocking, W. K.: The spaced antenna drift method, MAP Handbook, 9, 171-186, 1983b.

Hocking, W. K.: Radar studies of small scale structure in the upper middle atmosphere and lower ionosphere, Adv. Space Res., 7, 10327-10338, 1987.

Hocking, W. K.: Target parameter estimation, MAP Handbook, 30, 228-268, 1989.
Hocking, W. K., May, P., and Röttger, J.: Interpretation, reliability, and accuracies of parameters deduced by the spaced antenna method in middle atmosphere applications, PAGEOPH, 30, 571604, 1989

Holdsworth, D. A. and Reid, I. M.: A simple model of atmospheric radar backscatter: Description and application to the full correlation analysis of spaced antenna data, Radio Sci., 30, 1263-1280, 1995.

Holdsworth, D. A. and Reid, I. M.: An investigation of biases in the full correlation analysis technique, Adv. Space Res., 20, 12691272, 1997.

Holloway, C. L., Doviak, R. J., and Cohn, S. A.: Cross correlations of fields scattered by horizontally anisotropic refractive index irregularities, Radio Sci., 32, 1911-1920, 1997a.

Holloway, C. L., Doviak, R. J., Cohn, S. A., Lataitis, R. J., and Van Baelen, J. S. (HDC): Cross correlations and cross spectra for spaced antenna wind profilers, 2, Algorithms to estimate wind and turbulence, Radio Sci., 32, 967-982, $1997 \mathrm{~b}$.

Hysell, D. L., Yamamoto, M., and Fukao, S.: Imaging radar observations and theory of type I and type II quasi-periodic echoes, J. Geophys. Res. - Space Phys. 107 (A11), Art. No. 1360, 2002.

Ishimaru, A.: Wave Propagation and Scattering in Random Media, Oxford Univ. Press, 1997.

Kishore, P., Reddy, K. K., Rao, D. N., Rao, P. B., Jain, A. R., Rama, G. V., and Sankar, S.: A statistical comparison of Indian ST radar and rawinsonde wind measurements, Indian J. radio Space Phys., 29, 102-114, 2000

Larsen, M. F. and Röttger, J.: The spaced antenna technique for radar wind profiling, J. Atmos. Oceanic Technol., 6, 920-938, 1989.

Lataitis, R. J., Clifford, S. F., and Holloway, C. L.: An alternative method for inferring winds from spaced-antenna radar measurements, Radio Sci., 30, 463-474, 1995.

Liu, C. H., Röttger, J., Pan, C. J., and Franke, S. J.: A model for spaced antenna observational mode for MST radars, Radio Sci., 25, 551-563, 1990

Luce, H., Fukao, S., Yamamoto, M., Sidi, C., and Dalaudier, F. Validation of winds measured by MU radar with GPS radiosondes during the MUTSI campaign, J. Atmos. Oceanic Technol., $18,817-829,2001$

Luce, H., Röttger, J., Crochet, M., Hanuise, C., Yamamoto, M., and Fukao, S.: On the interpretation of the layered structures detected by mesosphere-stratosphere-troposphere radars in dual frequency domain interferometry mode, Radio Sci., 34, 1077$1083,1999$.

Luce, H., Röttger, J., Crochet, M., Yamamoto, M., and Fukao, S.: Scattering layer thickness and position estimated by radar frequency domain interferometry, 2. Effects of tilts of the scattering layer or radar beam, Radio Sci., 35, 1109-1127, 2000.

Manson, A. H., Gregory, J. B., and Stephenson, D. G.: Winds and wave motions to $110 \mathrm{~km}$ at mid-latitudes, I, Partial reflection radio wave soundings, J. Atmos. Sci., 31, 2207-2215, 1974.

Meek, C. E.: An efficient method for analyzing ionospheric drifts data, J. Atmos. Terr. Phys., 42, 835-839, 1980a.

Meek, C. E.: Triangle size effect in spaced antenna wind measurements, Radio Sci., 25, 641-648, 1980 b.

Meek, C. E. and Manson, A.: MF radar spaced antenna experiment: wind variance vs. record length, J. Atmos. Solar-Terr. Phys., 63 , 181-191, 2001

Migdal, A. B.: Qualitative Methods in Quantum Theory, W. A. Benjamin Inc., 1977.

Monin, A. S. and Yaglom, A. M.: Statistical Fluid Mechanics: Me- 
chanics of Turbulence, Vol. 2, MIT Press, 1975.

Palmer, R. D.: Multiple-receiver techniques for atmospheric wind profiling, IEEE Geosci. Remote Sens. Soc. Newsl., 9-17, 1994.

Palmer, R. D., Larsen, M. F., and Sheppard, E. L.: Poststatistic steering wind estimation in the troposphere and lower stratosphere, Radio Sci., 28, 261-271, 1993.

Palmer, R. D., Woodman, R. F., Fukao, S., Larsen, M. F., Yamamoto, M., Tsuda, T., and Kato, S.: Frequency domain interferometry observations of tropo/stratospheric scattering layers using the MU radar: Description and first results, Geophys. Res. Lett., 17, 2189-2192, 1990a.

Palmer, R. D., Woodman, R. F., Fukao, S., Tsuda, T., and Kato, S.: Three-antenna poststatistic steering using the MU radar, Radio Sci., 25, 1105-1110, 1990b.

Praskovskaya, E., Praskovsky, A., Chen, J.-S., and Chu, Y.-H. Wind measurements by the Chung-Li radar in the presence of strong clutter and hard targets, Proc. of the 10th Workshop on Technical and Scientific Aspects of MST radar, Piura, Peru, May 1320, 2003, Published by Radio Observatorio de Jicamarca, Lima, Peru, 230-233, 2003.

Praskovsky, A. A. and Praskovskaya, E. A. (PPa): Structurefunction-based approach to analyzing received signals for spaced antenna radars, Radio Sci., 38(4), 7-1-7-25, 2003 a.

Praskovsky, A. and Praskovskaya, E. (PPb): Towards the advanced measurements of atmospheric turbulence by spaced antenna radars, Proc. of the 10th Workshop on Technical and Scientific Aspects of MST radar, Piura, Peru, May 13-20, 2003, Published by Radio Observatorio de Jicamarca, Lima, Peru, 302305, 2003b.

Riggin, D. M., Fritts, D. C., Tsuda, T., Nakamura, T., and Vincent, R. A.: Radar observation of a 3-day Kelvin wave in the equatorial mesosphere, J. Geophys. Res., 102, 26 141-26 158, 1997.

Röttger, J.: Investigations of the lower and middle atmosphere dynamics with spaced antenna drift radar, J. Atmos. Terr. Phys., 43, 277-292, 1981.

Röttger, J. and Vincent, R. A.: VHF radar studies of tropospheric velocities and irregularities using spaced antenna techniques, Geophys. Res. Lett., 5, 917-920, 1978.

Sheppard, E. L., Larsen, M. F., Palmer, R. D., Fukao, S., Yamamoto, M., Tsuda, T., and Kato, S.: A statistical comparison of spaced antenna and spatial interferometry wind estimation, Radio Sci., 28, 585-593, 1993.

Stankov, B. B., Gossard, E. E., Weber, B. L., Lataitis, R. J., White, A. B., Wolfe, D. E., and Welsh, D. C.: Humidity gradient pro- files from wind profiling radars using the NOAA/ETL advanced signal processing system (SPS), J. Atmos. Oceanic Technol., 20, 3-22, 2003.

Steinhagen, H., Christoph, A., Chechowsky, P., Görsdorf, U., GubeLenhardt, M., Lippmann, J., Neisser, J., Rüster, R., Schmidt, G., Wergen, W., and Yoe, J. G.: Field campaign for the comparison of SOUSY radar wind measurements with rawinsonde and model data, Ann. Geophys., 12, 746-764, 1994.

Tatarskii, V. I.: The Effects of the Turbulent Atmosphere on Wave Propagation, UDC 551.510, U.S. Dep. of Commerce, Washington, D.C., 1971.

Thomas, L., Astin, I., and Worthington, R. M.: A statistical study of underestimates of wind speeds by VHF radar, Ann. Geophys. $15,805-812,1997$.

Townsend, A. A.: The Structure of Turbulent Shear Flow, Cambridge Univ. Press, 1956.

Tsuda, T., Sato, T., Hirose, K., Fukao, S., and Kato, S.: MU radar observations of the aspect sensitivity of backscattered VHF echo power in ther troposphere and the lower stratosphere, Radio Sci., 21, 971-980, 1986.

Tsuda, T., Van Zandt, T. E., and Saito, H.: Zenith-angle dependence of VHF specular relection echoes in the lower atmosphere, J. Atmos. Terr. Phys., 59, 761-775, 1997.

Tsuda, T., Yamamoto, M., Sato, T., and Kato, S.: Comparison between MU radar and the Kyoto meteor radar, Radio Sci., 20, 1241-1246, 1985.

Van Baelen, J. S., Tsuda, T., Richmond, A. D., Avery, S. K., Kato, S., Fukao, S., and Yamamoto, M.: Comparison of VHF Doppler beam swinging and spaced antenna observations with the MU radar: First results, Radio Sci., 25, 629-640, 1990.

Vincent, R. A.: Relationship of spaced antenna and Doppler techniques for velocity measurements, MAP Handbook, 14, 126130, 1984.

Vincent, R. A., May, P. T., Hocking, W. K., Elford, W. G., Candy, B. H., and Briggs, B. H.: First results with Adelaide VHF radar: Spaced antenna studies of winds, J. Atmos. Terr. Phys., 40, 353 366, 1987.

Vincent, R. A. and Röttger, J.: Spaced antenna VHF radar observations of tropospheric velocities and irregularities, Radio Sci., 15, 319-335, 1980.

Zhang, G., Doviak, R. J., Vivekanandan, J., Brown, W. O. J., and Cohn, S. A.: Cross-correlation ratio method to estimate crossbeam wind and comparison with a full correlation analysis, Radio Sci., 38, 8052-8063, 2003. 